Development of a multiparametric characterisation protocol for chert investigation and application on the Gargano Promontory mines

Emanuela Delluniversità, Italo Maria Muntoni, Ignazio Allegretta, Massimo Tarantini, Alessandro Monno, Patrizia Maiorano, et al.

Archaeological and Anthropological Sciences

ISSN 1866-9557

Archaeol Anthropol Sci

DOI 10.1007/s12520-019-00875-8

\section{ONLINE FIRST}

Archaeological and Anthropological Sciences

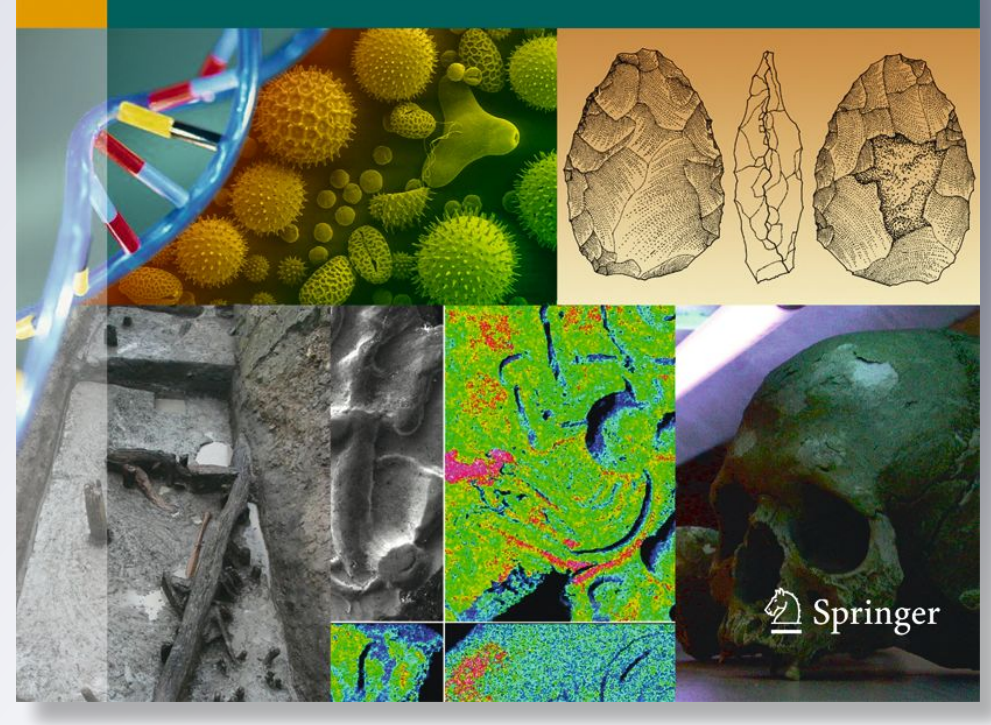

包 Springer 
Your article is protected by copyright and all rights are held exclusively by SpringerVerlag GmbH Germany, part of Springer Nature. This e-offprint is for personal use only and shall not be self-archived in electronic repositories. If you wish to self-archive your article, please use the accepted manuscript version for posting on your own website. You may further deposit the accepted manuscript version in any repository, provided it is only made publicly available 12 months after official publication or later and provided acknowledgement is given to the original source of publication and a link is inserted to the published article on Springer's website. The link must be accompanied by the following text: "The final publication is available at link.springer.com". 


\title{
Development of a multiparametric characterisation protocol for chert investigation and application on the Gargano Promontory mines
}

\author{
Emanuela Delluniversità ${ }^{1}$ (D) Italo Maria Muntoni $^{2} \cdot$ Ignazio Allegretta $^{3} \cdot$ Massimo Tarantini $^{4} \cdot$ Alessandro Monno $^{1}$. \\ Patrizia Maiorano ${ }^{1} \cdot$ Angela Girone $^{1} \cdot$ Michele Morsilli $^{5} \cdot$ Roberto Terzano $^{3} \cdot$ Giacomo Eramo $^{1}$
}

Received: 30 January 2019 / Accepted: 27 May 2019

(C) Springer-Verlag GmbH Germany, part of Springer Nature 2019

\begin{abstract}
A nondestructive multiparametric protocol for chert investigations (NM-PCI) was developed to provide objective criteria for raw material procurements and provenance investigations of archaeological cherts. For this purpose, macroscopic and mesoscopic characteristics (structures, textures and paleontological contents) were used to describe the main visible chert features as well as colourimetric and geochemical data acquired with portable devices. Data handling was performed by means of cluster analysis multivariate statistics, with an appropriate selection of algorithms to process and visualize mixed data matrices (partitioning around medoids (PAM), t-distributed stochastic neighbor embedding (t-SNE)). The protocol was applied to chert samples from the Gargano Promontory (Italy, Apulia) mining complex located in the districts of Peschici, Vieste and Mattinata. These mines, exploited for approximately four millennia, constitute an exceptional prehistoric record of mining archaeology and, above all, a key tool to understand the Neolithisation process throughout the Adriatic region. A map of the primary and secondary chert sources of the Gargano Promontory was produced to manage field sampling of mine structures. Waste materials were selected for the geologic samples of the five mines. Due to their inaccessibility, the samples from two mines consisted exclusively of waste materials. The NMPCI proved able to discriminate most of the analysed mines and is well suited to provenance investigations of archaeological lithic industries. Compared to previous geochemical studies, the nondestructive method proposed here improves geological resolution, allowing the discrimination of cherts within the same formation.
\end{abstract}

Keywords NM-PCI $\cdot$ Chert $\cdot$ Gargano Promontory $\cdot$ Neolithisation process $\cdot$ Prehistoric mines

Electronic supplementary material The online version of this article (https://doi.org/10.1007/s12520-019-00875-8) contains supplementary material, which is available to authorized users.

\author{
Giacomo Eramo \\ giacomo.eramo@uniba.it \\ Emanuela Delluniversità \\ emanuela.delluniversita@uniba.it \\ Italo Maria Muntoni \\ italomaria.muntoni@beniculturali.it \\ Ignazio Allegretta \\ ignazio.allegretta@uniba.it \\ Massimo Tarantini \\ massimo.tarantini@beniculturali.it \\ Alessandro Monno \\ alessandro.monno@uniba.it \\ Patrizia Maiorano \\ patrizia.maiorano@uniba.it \\ Angela Girone \\ angela.girone@uniba.it
}

\author{
Michele Morsilli \\ michele.morsilli@unife.it \\ Roberto Terzano \\ roberto.terzano@uniba.it
}

1

Dipartimento di Scienze della Terra e Geoambientali, Università degli Studi di Bari “A. Moro", Via Orabona 4, 70125 Bari, Italy

2 Soprintendenza Archeologia, Belle Arti e Paesaggio per le Province di Barletta-Andria-Trani e Foggia, Via A.A. Valentini 8, 71121 Foggia, Italy

3 Dipartimento di Scienze del Suolo, della Pianta e degli Alimenti, Università degli Studi di Bari “A. Moro”, Via Orabona 4, 70125 Bari, Italy

4 Soprintendenza Archeologia Belle Arti e Paesaggio per la Città metropolitana di Firenze, Piazza de' Pitti 1, 50125 Florence, Italy

5 Dipartimento di Fisica e Scienze della Terra, Università degli Studi di Ferrara, Via Saragat 1, 44122 Ferrara, Italy 


\section{Introduction}

Prehistoric studies about the cultural and technological transformation of lithic industries constitute a key means of exploring many archaeological issues (e.g. Ambrose 2001; Pelegrin and Roche 2017). Among these lithic resources, chert is one of the most widely employed raw materials for artifact manufacture throughout prehistory and is commonly indicated in the archaeological literature as both flint and chert. Furthermore, prehistoric people must have been intensely interested in chert sources and in other siliceous rocks (e.g. Allard et al. 2008; Binder et al. 1990; Bressy 2003; Léa 2004; Perlès 2004; Korlin and Weisgerber 2006 and the Proceedings of the International Symposiums on Knappable Materials held at Iaşi in 2013 and at Barcelona in 2015 edited in the Journal of Lithic Studies 2014 (1:1) and 2016 (3:2), respectively). Lithic resources are essential to many archaeologists attempting to understand past cultures from a sociocultural perspective.

Since lithic artifacts were produced almost exclusively by mechanical modification, no compositional change is assumed between the source and the finished artifact. Although stone can be considered the ideal material for provenance studies, the identification of geological sources for chert artifacts is a difficult task. Flaked stone artifacts are among the most common finds in the archaeological record and are often made from materials that do not occur locally (e.g. obsidian and chert). They are the product of several distinct actions performed at different times and locations consisting of the procurement of the raw material, preparation of a core, chipping, use and retouching (Tykot 2004).

Unlike obsidian and other materials with little visual variability, cherts from different sources are often visually distinctive; thus, visual inspection alone has been used by archaeologists as a moderate identification technique since the late nineteenth century (e.g. Damour 1865, de Chasteignier 1868). In more recent times, Luedtke (1979) has stressed that visual attributes should not be overlooked and should be objectively determined and quantified so that identifications can be clearly assessed by other scholars. Relative to obsidian, chert is also a challenge for geochemists because it essentially contains only silicon and oxygen and shows low to very low concentrations of other elements (Gauthier et al. 2012).

Macroscopic descriptions of chert lack objectivity and standardisation in many archaeological reports. Resulting data are often plagued by poor reliability and reproducibility, and they do not allow easy statistical analysis. Archaeologists and researchers attempted to adopt standardized methods of analysis for geological chert and artifacts as early as the first half of the twentieth century (e.g. Saint-Venant, de Saint-Venant 1911; Deflandre 1935; Brajnikov 1937: Krukowski 1939; Folk and Weaver 1952; Valensi 1960). Although new analytical methods were adopted in later times (see below), the issue of the terminology, the analytical protocols and the compositional reference groups persists (Turq 2005; Shackley 2008).

A real terminology issue occurs regarding fine-grained siliceous sedimentary rocks. The term flint is frequently used both as a synonym and as a varietal name for chert (Tucker 2001; Boggs 2009). Normally, British geologists employed the flint term to indicate a particular type of chert characterized by a very fine-grained (mostly black nodular chert) common in Cretaceous chalks; meanwhile, the term chert referred to the light-coloured, impure materials found elsewhere as nodules in limestones or in beds among shales. In contrast, American geologists used the word chert as the general term for all sedimentary rocks composed primarily of microcrystalline quartz and considered all other terms, such as flint, jasper, novaculite and porcelanite, to be varieties of chert (Luedtke 1992). Usually, in the geological record, cherty rocks are subdivided into bedded types resulting from primary accumulation (e.g. radiolarites and diatomites) and the nodular type of diagenetic origin (Tucker 2001; Greensmith 2012). For clarity, the term chert is adopted in this work as a generic group name used for fine-grained siliceous sedimentary rocks in accordance with the sensu lato of Tucker (2001) because of the lithological variety of the analysed materials.

One of the phenomena involved in the so-called Neolithisation process is the exploitation of chert mines (for a recent chronological overview, see Consuegra and Díaz-del-Río 2018). In the Apulian Region, important evidence for the Neolithisation process was found in 1981 with the 'Defensola A' mine discovery (Galiberti 2005). Subsequently, a mining archaeology project was carried out in the Gargano area, leading to the detection of a large complex of at least 20 mining sites concentrated in the northeastern part of the promontory (Basili et al. 1995; Tarantini 2005; Tarantini et al. 2011; Tarantini and Galiberti 2011). This mining complex was active from the early Neolithic (sixth millennium BCE) to the early Bronze Age (second millennium BCE). The mining activities carried out over four millennia, together with the large size of some mines and the extraction skills required, contribute to making Gargano one of the main sites of chert supply in the central-northern Mediterranean area.

The method proposed in this study aims to investigate a variety of features (macroscopic to microscopic, colourimetric and geochemical) on hand specimens and archaeological artifacts and to improve traditional approaches (visual comparison) in which the high similarities of chert raw materials led to inaccurate attributions and provenances.

The archaeological issue of chert sourcing from the mines of Gargano is considered as a case study to test the nondestructive multiparametric protocol for chert investigations 
(NM-PCI), primarily proposed by Tarantini et al. (2016) and here opportunely revisited. Consequently, the main goal of this paper is to propose a standardised procedure that uses a mixed data matrix obtained from macroscopic to microscopic descriptions and from portable spectroscopic devices to run multivariate statistics for classification and inferences.

\section{Chert sourcing: state of the art}

As mentioned above, early works carried out to identify chert sources date back to the end of the nineteenth century and were essentially based on macroscopic description (i.e. colour, morphoscopy, micropaleontology, petrography and physical parameters) and only later was optical microscopy introduced (Fernandes 2012). In the 1970s, increased attention to sedimentary siliceous materials brought new methodological approaches. The first studies highlighted the importance of lithic sourcing to retrace prehistoric connection networks (Sieveking et al. 1972; Luedtke 1978). These investigations aimed at recognising and differentiating specific geological chert sources and successively comparing archaeological artifacts to their resources (Sieveking et al. 1972; Luedtke 1979; Selivanova 1984; Shackley 2008).

In the field of archaeology, a macroscopic approach is commonly employed to describe visible properties of artifacts and to compare them with geological samples. Several authors have proposed different analytical strategies in order to identify key features of the chert artifacts useful to their sourcing. The identification of chert raw materials is a geological issue that has been approached through either geochemistry or petrography (e.g. Sieveking et al. 1972; Séronie-Vivien and Seronie-Vivien 1987; Bressy 2002; Fernandes and Raynal 2006; Tarantini et al. 2016).

Variations in geochemical patterns in cherty limestones of different geographical areas have been observed by Sieveking et al. (1970, 1972), who carried out quantitative analyses on sources from Great Britain and Western Europe by means of atomic emission spectroscopy (AES) and atomic absorption spectroscopy (AAS). These studies showed that the variations in the trace element contents of chert were mainly related to variations in the noncarbonate materials (clay and phosphate minerals, organic matter) and that the geochemical variations in certain trace elements were a function of the depositional environment of the chalk in which the nodules formed. The same chert artifacts used by Sieveking (Sieveking et al. 1972) were subjected to neutron activation analysis (NAA) by Aspinall and Feather (1972) to characterize prehistoric chert mining sites. Their research highlights the wide variations in certain elements and the limits of provenance studies dictated by geological variability.
Luedtke $(1978,1979)$ addressed the problem of trace element variation within geologic sources of chert and the extent to which chemical variation may be congruent with visual variation, with reference to colour, texture and structure. Luedtke (1979) used the term 'source' in a broad sense to indicate the area from which the chert was originally obtained as raw material, including primary sources (bedrock deposits) and secondary sources (glacial, stream, beach and talus slope deposits). The chemical variability existing within formations contradicts the assumption that chert-bearing formations can be considered chemically homogeneous, and systematic sampling procedures must be used to characterize each source. The understanding of the variations within and between sources before the attribution of artifacts is of paramount importance. The results obtained in these studies suggest that trace element analysis is a useful tool in archaeology, but when the sources are very close in time and space, they can be chemically similar and therefore scarcely distinguishable.

The monograph edited by Sieveking and Hart (1986) provided a collection of specialized studies that represented a step forward in the scientific investigation of chert, where different contributions from both Earth scientists and archaeologists contributed to defining a common ground in this research field. The geological approach of Séronie-Vivien (Séronie-Vivien and SeronieVivien 1987), applied to the chert of the North Aquitaine carbonate platform, showed that the morphology and textures of chert were connected to the sedimentary environment. As the silicification process frequently preserves the original textural characteristics, Dunham's classification (Dunham 1962) was applied to describe the textural properties of chert nodules. This work initially introduced in prehistoric research a methodological approach based on geological terminology to describe chert samples on the basis of macroscopic and microscopic observations. Microfacies of rocks and micropaleontological contents were identified, and the combination of features allowed researchers to reconstruct the depositional environments. However, even if the use of Dunham's (1962) classification for limestone texture is quite widespread in chert petrography (e.g. Séronie-Vivien and Seronie-Vivien 1987; Bressy 2002; Fernandes and Raynal 2006), note that its petrological meaning is not the same. In the case of chert, the diagenetic processes and the possible mobilisation of the sediment (e.g. bioturbation, slumping) can yield significant modifications in the chert textures compared to the original ones, as present in the parent sediment (more on this topic in $\S$ 5.1). The petrographic-mineralogical protocol proposed by Pawlikowski (1989) aimed to standardize petrological investigations of raw materials: the procedure suggested a macroscopic description that includes determining the colour of the samples by the Munsell colour chart 
(Munsell 1915), the luster according to mineralogical terms and definitions of transparency and fissility. Furthermore, a microscopic description was carried out on thin sections, describing texture, structure and mineral composition. These three contributions represent the fundamentals of the archaeometric study of chert because they propose a standardisation of features frequently considered subjective and not codified. Sarabia Rogina (1990) proposed a similar approach, with macroscopic descriptions based on the geological age of the formations, the colour (as measured with the code by Cailleux and Taylor (1963)), the texture (as a function of touch and grain size) and the luster (discerned in category), type and thickness of the cortex.

An important work on chert from different points of view is certainly that of Luedtke (1992). More specifically, her research suggests a standard description of visible properties in order to facilitate comparisons using formalized descriptions of geological, mineralogical and petrological features. This study proposed for the first time a protocol for chert macroscopic description that involved the colour measure by the Munsell colour chart as a more accurate method to evaluate the chromatic features of chert. A method to measure the translucency of chert was proposed by Ahler (1983), although this visible property varies with the material's thickness. Luster is defined with qualitative terms, but Luedtke (1992) remarked the relevance of quantifying luster as an expression of both mineralogy and surface characteristics of a material. The observations of fracture surfaces (that could appear uneven, rough or smooth) are strictly connected with textural properties that are a function of porosity, the presence of inclusions and microcracks around the grains. The gradual or abrupt variations in structure are defined with an appropriate terminology distinguishing between replacement and diagenetic features. Luedtke (1992) pointed out two possible outer layers; she defined cortex as only the outer layer that developed during the diagenetic process and recommended the term 'weathering rind' in the case of the weathering process. This study proceeded by examining chert under a light microscope and a scanning electron microscope. The information was collected at different scales, and correlations resulted between luster and texture (that provided information about grain size) and between fracture surfaces and colour, frequently linked to occurrence of impurities. Luedtke (1992) argued that interrelations between various properties of chert allowed predicting some of the properties on the basis of the others. Although this protocol improved the archaeological practice with more reproducible observations and a suitable terminology, some features remained subjective (i.e. colour and luster).
Hess (1996) highlighted that petrographic and geochemical signatures were essential in identifying the provenance of chert artifacts. Hess (1996) defined 'source' as any outcrop of potentially useful lithic raw material and 'quarry' as only outcrops exploited during prehistoric time that show visible signs of extraction. A source may be extremely variable in elemental composition; therefore, chert provenance analysis based on geochemistry is very difficult. Thus, changes in trace element concentrations are likely to occur due to weathering and heat treatments. Macroscopic and microscopic attributes as well as elemental concentrations were analysed by Hesse (Hess 1996). Macroscopic descriptions included colour, luster, fracture characteristics, light transmittance and the presence of every vein or inclusion. Based on Luedtke (1992), Hess described the nature of the deposit, the structure using Luedtke's terminology and for the first time the colour of the cortex measured with a Munsell chart as well as its variability on freshly fractured surfaces. Hess remarked that the use of petrographic and geochemical signatures for chert provenance analysis is unavoidable and demonstrated the limits of macroscopic examination. Macroscopic descriptions, as in Luedtke's study, showed observer subjectivity.

NAA and ICP-MS were used by Hess (1996) for chert provenance study in the Columbia Plateau, and the two techniques produced comparable results. The author suggested $\mathrm{X}$ ray fluorescence (XRF) as a third possible analytical option for chert sourcing and indicated discriminant analysis as the statistics best suited for large-scale provenance analysis.

The approach of Malyk-Selivanova et al. (1998) to chert sourcing proposed geological sampling and analysis by NAA for the detection of trace element concentrations and descriptive statistics to process chemical data. Unweathered and unpatinated parts of artifacts should be selected for chemical analysis. The author discerned between two indicators of provenance: $(i)$ 'key signatures', for the discrimination of cherts belonging to different stratigraphic formations or units (i.e. depositional environments), and (ii) diageneticmetamorphic signatures, useful to discriminate outcrops within the same stratigraphic unit (e.g. $\mathrm{Sr}, \mathrm{Rb}$ and $\mathrm{Ba}$ ).

Bressy (2002) discerned between 'primary sources', when chert is within the host rocks, and 'subprimary' and 'secondary chert sources', based on their depositional position and traces of mechanical transport on the surfaces. The macroscopic approach represents the core of Bressy's (2002) research method. She proposed empirical observations combined with nondestructive petrographic analyses of the bulk samples under a stereomicroscope. This approach is based on the observation of the frequency, dimension and nature of inorganic 
inclusions, oxides, organic matter and micropaleontological contents. A similar study used macroscopic descriptions following the terminology proposed by the CIRCALP project (Affolter et al. 1999), which aimed to describe chert systematically and was carried out on fresh surfaces of geological samples. A more detailed terminology is proposed to define the chemical nature of the cortex. Since cortex and cortex/chert transitions can be a peculiarity of chert in a primary source, a detailed description is needed (e.g. thickness, colour, type of transition). Moreover, the outer surface of secondary source chert is indicated as the 'neocortex'. The changes in the weathered surface are consequences of exposure, transport and climate. This method does not consider colour measurements and suggests a terminology to describe luster, fracture, patina and structures. Observations under a stereomicroscope complement the macroscopic descriptions, as micropaleontological contents may be visible and add useful discriminant information without destroying the sample; however, thin sectioning can be necessary to increase and clarify complex cases. Bressy (2002) also suggested applying the methods used for carbonate rocks, since cherty rocks result from diagenetic processes of carbonate rocks.

The observations with binocular loupes frequently allowed discernment of different microfacies. Textural properties were defined according to the Dunham classification (Dunham 1962). Furthermore, a detailed description of each inclusion within the matrix and their structures allowed recognition of sedimentary facies and, when possible, proposition of a paleoenvironmental interpretation.

Moreover, the need to better discriminate chert's age and facies found in the micropaleontological approach is an appropriate method, particularly relevant for archaeological studies (Bressy 2002). The first implementation of the method dates back to the 1980s from Masson's work (Masson 1981); subsequently, major developments were contributed by Affolter (1994, 2002) and Bressy (2002) for provenance studies. The speed of characterisation, nondestructivity and low cost of the methodology proposed by Bressy (2002) constituted fundamental progress in chert investigations. The disadvantages of this methodology are connected with the subjectivity of empirical descriptions and the impossibility of applying the method to patinated pieces. In Italy, this method has been used by Bertola $(2011,2012)$ for chert procurement in the Val di Non formations (Trentino, Italy).

Fernandes and Raynal (2006) retraced the complexity of occurrences observable on the surfaces of prehistoric artifacts at different scales using a petroarchaeological method in order to identify the sources of raw materials and the locations of deposition of the objects analysed. The method, which includes mineralogical, petrographical, micropaleontological and morphological approaches, aimed at reconstructing 'silica evolutionary chains', produced by genetic and postgenetic phenomena. The examination of the micromorphology of different types of neocortex (e.g. colluvium, recent or old alluvium) allowed them to recognize the features of each geological environment. The so-called 'predepositional phase of the evolutionary chain' explained the palaeogeographic position of chert before prehistoric modifications (i.e. primary, subprimary or secondary). Natural and anthropic surface features were detected using optical and scanning electron microscopy to characterize internal, endocortical and cortical portions. Fernandes and Raynal (2010) remarked that the internal zone is less subject to modifications compared to the more external portions.

Three possible textures (unimodal, bimodal and trimodal) were distinguished. Thus, it was possible to build an inventory of regional resources useful for comparing lithic artifacts with cherts from primary, subprimary or secondary deposits.

The nondestructive technique of energy-dispersive $\mathrm{X}$ ray fluorescence (ED-XRF), previously applied for the chemical characterisation of obsidian artifacts (Hughes 1983, 1988; James et al. 1996; Shackley 1988, 1995), was adopted by Gauthier et al. (2012) to determine Canadian chert sourcing. To identify lithic procurement of Magdalenian Pyrenean sites, Sánchez de la Torre et al. (2017) carried out a geochemical characterisation of two chert formations in the Pyrenees, which showed similar textural and micropaleontological features, using ED-XRF and laser ablation inductively coupled plasma mass spectrometry (LA-ICP-MS) and found differences between the trace element concentrations detected with the two instruments.

In the last decade, portable X-ray fluorescence (pXRF) devices have been widely adopted in order to chemically characterize many archaeological materials (in the field and in the laboratory), including obsidian (Craig et al. 2007; De Francesco et al. 2008; Nazaroff et al. 2010; Sheppard et al. 2011; Forster and Grave 2012; Glascock 2012).

Although incomplete, this overview of the analytical strategies shows that a single methodology of general validity and acceptance by the scientific community, which is can provide accurate and precise data via preferably nondestructive investigation, is not yet available. A promising way to overcome the partial information derived from the single analytical techniques may be to merge together the obtained data in a multivariate statistical treatment to come closer to the real complexity of the material, as attempted in this work. 


\section{The mining complex of the Gargano Promontory}

Deep mining evidence on the Gargano Promontory represents exceptional proof of the ancient Neolithic populations in the southern part of Italy (Muntoni 2012). The plethora of mining sites shows the ancient systematic exploitation of chert, thus highlighting the importance of the Gargano area in the trade of raw materials during Neolithic phases (Di Lernia et al. 1997). From the earliest discoveries of mining structures performed by Rellini in the 1930s (Rellini et al. 1930-1931), several surveys have revealed many mining complexes distributed in the northeastern part of the promontory, in particular the Defensola mine, exploited since the beginning of the Neolithic (Galiberti 2005). Systematic mining research has been launched with the 'Gargano Prehistoric Flint Mines Project' (Di Lernia et al. 1990), which aimed to examine the mining phenomenon in the area in its entirety, historical and cultural implications included. A more detailed survey was conducted in 2005 by Tarantini (PhD thesis), who produced the most recent catalog of the prehistoric Gargano mines. The catalog collects and contextualizes all the known mine sites in this area, both geographically and chronologically (Tarantini and Galiberti 2011).

The mining network was active from the early Neolithic to the early Bronze Age. Radiometric dating of Defensola A indicates that this site is the most ancient in the area (cal. $2 \sigma$ 6010-5727 BCE) and in addition corresponds to the beginning of the Neolithisation process in the southeastern part of the Italian peninsula. Conversely, the most recent date was obtained for the Cruci mine, active until the ancient Bronze Age (Tarantini et al. 2011; Tarantini and Galiberti 2011).

The mining activities carried out over four millennia, the large size of some mines and the skills required identified Gargano as the main area of chert supply in the centralnorthern Mediterranean region (Tarantini et al. 2016).

\section{Geological setting}

The Gargano Promontory belongs to the Apulia carbonate platform (ACP) and is mostly composed of thick layers of shallow-water carbonate and their respective slopes to basin counterparts formed between the Late Jurassic and Eocene (Bernoulli 1972; Bosellini et al. 1999; Borgomano 2000; Morsilli et al. 2004; Morsilli et al. 2017). The ACP represents a main paleogeographic element of the southern border of the Mesozoic Tethys Ocean. Due to its geological setting, this platform, as well as other peri-Adriatic platforms, is compared with the Bahamas Bank in terms of facies and size (Eberli et al. 1993; Bosellini et al. 1999). Moreover, this longlasting carbonate succession, which is actually part of the undeformed foreland of the Southern Apennine thrust belt, is delimited on both sides by basinal deposits. Apennine thrust sheets cover the western margin, while the eastern margin crops out in the Gargano Promontory and in the Maiella Mountain and passes to the basinal successions of the Adriatic to Ionian Basins (Zappaterra 1994; Cobianchi et al. 1997). The Jurassic-Cretaceous sequences accumulated in distinct depositional environments from shallow-water facies of the inner platform and margin to the progressively deeper water successions that belong to the slope and basin settings (Bosellini and Morsilli 1997; Morsilli and Bosellini 1997; Bosellini et al. 1999; Borgomano 2000; Morsilli et al. 2017). Eocene slope to basin facies are located mainly in the northeastern part of the Gargano Promontory between Peschici and Vieste and in a small outcrop near Mattinata (Fig. 1).

Numerous slope and basinal units include chert layers or nodules, but only a few exhibit evidence of prehistoric mining (e.g. Tarantini and Galiberti 2011). More specifically, chert layers and nodules are very abundant in the Upper Jurassic-Lower Cretaceous basinal limestones of the Maiolica Fm. (Tithonian-Aptian p.p.) and the Upper Cretaceous-Paleogene Scaglia Fm. (Cenomanian to Danian). Scattered layers are also present in the Lower Cretaceous Marne a Fucoidi Fm. (Aptian p.p.-Albian) and in the Eocene slope to baseof-slope succession of the Peschici Fm. Apart from the mining sites discovered recently, chert layers also occur in other Cretaceous stratigraphic units, such as the Ripe Rosse, Mattinata, Monte Sant'Angelo and Monte Acuto Limestones, accumulated in slope and base-of-slope settings (Fig. 2).

According to the most recent studies (Morsilli 2011, 2016), a map of the primary and secondary chert sources of the Gargano Promontory was prepared, showing only the formations targeted by chert mining and sampling (Fig. 1).

A first attempt to characterize the geological cherts from northeastern Gargano with petrographic (OM, SEM), mineralogical (XRPD) and geochemical methods (XRF and ICP-MS) was made by Gelato (1994). Subsequently, the cherts from the mines of the Gargano Promontory were studied by the research team led by Alberto M. Palmieri (D'Ottavio et al. 2000; D'Ottavio 2001; Volterra et al. 2002; D'Ottavio and Palmieri 2005), who tried to chemically characterize each mine through ICP-AES analyses. The results of these studies are compared to the results of the NMPCI method ( $(5)$. A more accurate allocation into geological formations is made in the case of Valle Guariglia I and Tagliacantoni (attributed respectively to the Scaglia Fm. and Peschici Fm. from bibliographic sources). The following discussion ( $(7)$ expands upon these issues, and the revised geological information can be found in Table 1. Electronic supplementary material (S1) includes data resulting from descriptions and measures from the NM-PCI method. 


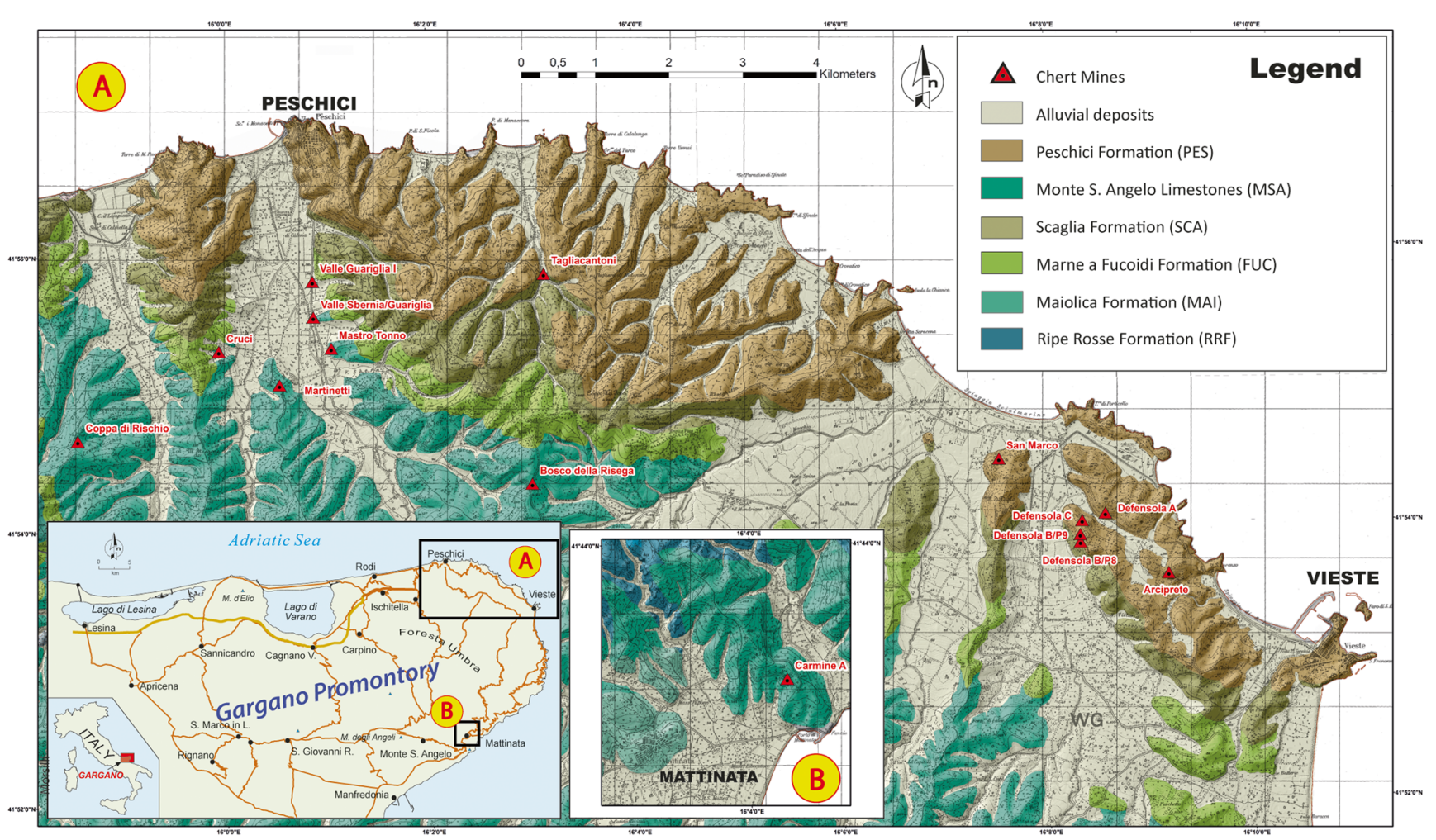

Fig. 1 Simplified geological map of the Gargano promontory and position of the chert mines here investigated (mod. after Morsilli 2016)

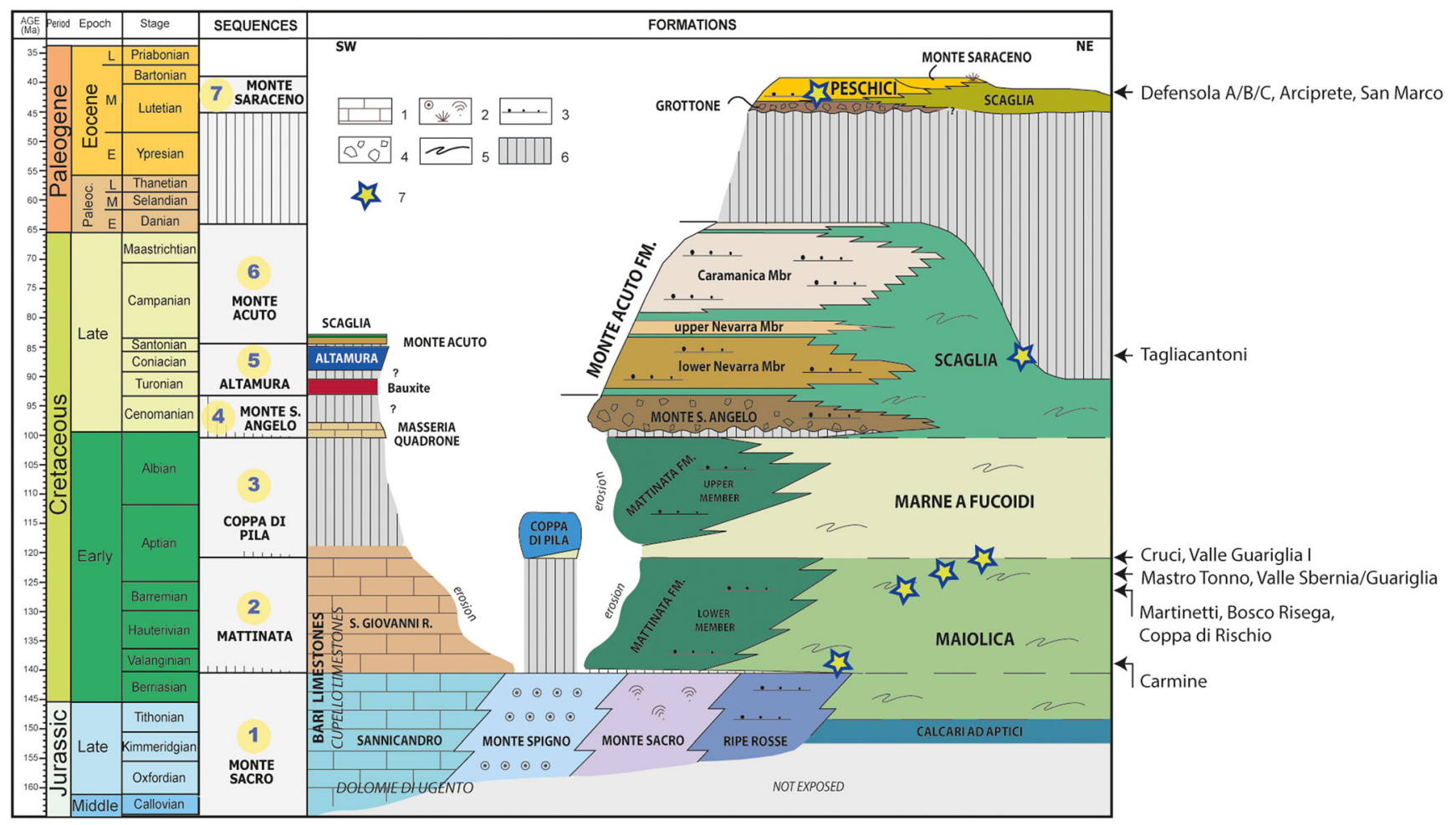

Fig. 2 Chronostratigraphic scheme of the Jurassic to Eocene stratigraphic units cropping out in the Gargano Promontory. 1, Inner Platform facies; 2, Marginal facies; 3 and 4, slope to base of slope facies; 5 , basinal facies; 6 , hiatus; 7 , position of chert layers and nodules associated to the mines here investigated (after Morsilli et al. 2017) 
Table 1 Summary of the archaeological and geological data of the analysed samples

\begin{tabular}{|c|c|c|c|c|c|c|c|}
\hline Sample & Mine & Type & Mine chronology & Formation & COOR_E & COOR_N & Position (shape) \\
\hline ARC MIN 1 & Arciprete & Waste & Early Neolithic & Peschici & $595,639.20$ & $4,638,617.73$ & - \\
\hline ARC MIN 2 & Arciprete & Waste & Early Neolithic & Peschici & $595,639.20$ & $4,638,617.73$ & - \\
\hline ARC MIN 3 & Arciprete & Waste & Early Neolithic & Peschici & $595,639.20$ & $4,638,617.73$ & - \\
\hline ARC MIN 4 & Arciprete & Waste & Early Neolithic & Peschici & $595,639.20$ & $4,638,617.73$ & - \\
\hline ARC MIN 6 & Arciprete & Waste & Early Neolithic & Peschici & $595,639.20$ & $4,638,617.73$ & - \\
\hline ARC MIN 7 & Arciprete & Waste & Early Neolithic & Peschici & $595,639.20$ & $4,638,617.73$ & - \\
\hline ARC sg18 & Arciprete & Waste & Early Neolithic & Peschici & $595,639.20$ & $4,638,617.73$ & - \\
\hline ARC sg19 & Arciprete & Waste & Early Neolithic & Peschici & $595,639.20$ & $4,638,617.73$ & - \\
\hline ARC 30_A & Arciprete & Waste & Early Neolithic & Peschici & $595,639.20$ & $4,638,617.73$ & - \\
\hline ARC 30_C & Arciprete & Waste & Early Neolithic & Peschici & $595,639.20$ & $4,638,617.73$ & - \\
\hline DEF A/1 & Defensola A & Geologic & Early and middle Neolithic & Peschici & $594,782.93$ & $4,639,419.16$ & - \\
\hline DEF A/3 & Defensola A & Geologic & Early and middle Neolithic & Peschici & $594,782.93$ & $4,639,419.16$ & - \\
\hline DEF A/1 30_A & Defensola A & Waste & Early and middle Neolithic & Peschici & $594,782.93$ & $4,639,419.16$ & - \\
\hline DEF A/1 30_B & Defensola A & Waste & Early and middle Neolithic & Peschici & $594,782.93$ & $4,639,419.16$ & - \\
\hline DEF A/1 30_C & Defensola A & Waste & Early and middle Neolithic & Peschici & $594,782.93$ & $4,639,419.16$ & - \\
\hline DEF A/1 30_D & Defensola A & Waste & Early and middle Neolithic & Peschici & $594,782.93$ & $4,639,419.16$ & - \\
\hline DEF B/P9_50 & Defensola B/P9 & Geologic & Neolithic/Copper Age & Peschici & $594,451.38$ & $4,639,145.16$ & Subprimary \\
\hline DEF B/P9_51 & Defensola B/P9 & Geologic & Neolithic/Copper Age & Peschici & $594,451.38$ & $4,639,145.16$ & Subprimary \\
\hline DEF B/P9_52 & Defensola B/P9 & Geologic & Neolithic/Copper Age & Peschici & $594,451.38$ & $4,639,145.16$ & Subprimary \\
\hline DEF B/P9_53 & Defensola B/P9 & Geologic & Neolithic/Copper Age & Peschici & $594,451.38$ & $4,639,145.16$ & Subprimary \\
\hline DEF B/P9_54 & Defensola B/P9 & Geologic & Neolithic/Copper Age & Peschici & $594,451.38$ & $4,639,145.16$ & Subprimary \\
\hline DEF B/P9_55 & Defensola B/P9 & Geologic & Neolithic/Copper Age & Peschici & $594,451.38$ & $4,639,145.16$ & Subprimary \\
\hline DEF B/P9_56 & Defensola B/P9 & Geologic & Neolithic/Copper Age & Peschici & $594,451.38$ & $4,639,145.16$ & Subprimary \\
\hline DEF B/P9_57 & Defensola B/P9 & Geologic & Neolithic/Copper Age & Peschici & $594,451.38$ & $4,639,145.16$ & Subprimary \\
\hline DEF B/P9_58 & Defensola B/P9 & Geologic & Neolithic/Copper Age & Peschici & $594,451.38$ & $4,639,145.16$ & Subprimary \\
\hline DEFB P9/1 & Defensola B/P9 & Waste & Neolithic/Copper Age & Peschici & $594,446.94$ & $4,639,131.01$ & - \\
\hline DEFB P9/2 & Defensola B/P9 & Waste & Neolithic/Copper Age & Peschici & $594,446.94$ & $4,639,131.01$ & - \\
\hline DEFB P9/3 & Defensola B/P9 & Waste & Neolithic/Copper Age & Peschici & $594,446.94$ & $4,639,131.01$ & - \\
\hline DEF B/P8/B_50a & Defensola B/P8 & Geologic & Neolithic/Copper Age & Peschici & $594,471.77$ & $4,639,006.97$ & Primary (nodule) \\
\hline DEF B/P8/B_52 & Defensola B/P8 & Geologic & Neolithic/Copper Age & Peschici & $594,471.77$ & $4,639,006.97$ & Primary (nodule) \\
\hline DEF B/P8/G_1a & Defensola B/P8 & Geologic & Neolithic/Copper Age & Peschici & $594,450.7$ & $4,639,031.21$ & Primary (nodule) \\
\hline DEF B/P8/G_2a & Defensola B/P8 & Geologic & Neolithic/Copper Age & Peschici & $594,450.7$ & $4,639,031.21$ & Primary (nodule) \\
\hline DEF B/P8/G_3a & Defensola B/P8 & Geologic & Neolithic/Copper Age & Peschici & $594,450.7$ & $4,639,031.21$ & Primary (nodule) \\
\hline Def B/P8/B_30_A & Defensola B/P8 & Waste & Neolithic/Copper Age & Peschici & $594,450.02$ & $4,639,029.91$ & - \\
\hline Def B/P8/B_30_B & Defensola B/P8 & Waste & Neolithic/Copper Age & Peschici & $594,450.02$ & $4,639,029.91$ & - \\
\hline Def B/P8/B_30_C & Defensola B/P8 & Waste & Neolithic/Copper Age & Peschici & $594,450.02$ & $4,639,029.91$ & - \\
\hline Def B/P8/B_30_D & Defensola B/P8 & Waste & Neolithic/Copper Age & Peschici & $594,450.02$ & $4,639,029.91$ & - \\
\hline Def B/P8/B_30_E & Defensola B/P8 & Waste & Neolithic/Copper Age & Peschici & $594,450.02$ & $4,639,029.91$ & - \\
\hline Def B/P8/B_30_G & Defensola B/P8 & Waste & Neolithic/Copper Age & Peschici & $594,450.02$ & $4,639,029.91$ & - \\
\hline Def B/P8/B_30_H & Defensola B/P8 & Waste & Neolithic/Copper Age & Peschici & $594,450.02$ & $4,639,029.91$ & - \\
\hline DEF C_1 & Defensola C & Geologic & Middle Neolithic & Peschici & $594,473.98$ & $4,639,325.04$ & Subprimary \\
\hline DEF C_2a & Defensola C & Geologic & Middle Neolithic & Peschici & $594,473.98$ & $4,639,325.04$ & Subprimary \\
\hline DEF C_3 & Defensola C & Geologic & Middle Neolithic & Peschici & $594,473.98$ & $4,639,325.04$ & Subprimary \\
\hline DEF C_4 & Defensola C & Geologic & Middle Neolithic & Peschici & $594,473.98$ & $4,639,325.04$ & Subprimary \\
\hline DEF C_5 & Defensola C & Geologic & Middle Neolithic & Peschici & $594,473.98$ & $4,639,325.04$ & Subprimary \\
\hline DEF C_6 & Defensola C & Geologic & Middle Neolithic & Peschici & $594,473.98$ & $4,639,325.04$ & Subprimary \\
\hline SM 50 & San Marco & Geologic & Middle Neolithic & Peschici & $593,334.31$ & $4,640,167.2$ & Primary (nodule) \\
\hline SM 51a & San Marco & Geologic & Middle Neolithic & Peschici & $593,334.31$ & $4,640,167.2$ & Primary (nodule) \\
\hline SM 52 & San Marco & Geologic & Middle Neolithic & Peschici & $593,334.31$ & $4,640,167.2$ & Primary (nodule) \\
\hline
\end{tabular}


Table 1 (continued)

\begin{tabular}{|c|c|c|c|c|c|c|c|}
\hline Sample & Mine & Type & Mine chronology & Formation & COOR_E & COOR_N & Position (shape) \\
\hline SM1 & San Marco & Waste & Middle Neolithic & Peschici & $593,349.00$ & $4,640,149.00$ & - \\
\hline SM2 & San Marco & Waste & Middle Neolithic & Peschici & $593,349.00$ & $4,640,149.00$ & - \\
\hline SM 30_A & San Marco & Waste & Middle Neolithic & Peschici & $593,349.00$ & $4,640,149.00$ & - \\
\hline SM 30_B & San Marco & Waste & Middle Neolithic & Peschici & $593,349.00$ & $4,640,149.00$ & - \\
\hline SM 30_C & San Marco & Waste & Middle Neolithic & Peschici & $593,349.00$ & $4,640,149.00$ & - \\
\hline SM 30_D & San Marco & Waste & Middle Neolithic & Peschici & $593,349.00$ & $4,640,149.00$ & - \\
\hline SM 30_E & San Marco & Waste & Middle Neolithic & Peschici & $593,349.00$ & $4,640,149.00$ & - \\
\hline SM 30_F & San Marco & Waste & Middle Neolithic & Peschici & $593,349.00$ & $4,640,149.00$ & - \\
\hline SM 30_G & San Marco & Waste & Middle Neolithic & Peschici & $593,349.00$ & $4,640,149.00$ & - \\
\hline VG 51 & Valle Guariglia I & Geologic & Recent Neolithic & Maiolica & $584,113.02$ & $4,642,513.99$ & Primary (nodule) \\
\hline VG 52a & Valle Guariglia I & Geologic & Recent Neolithic & Maiolica & $584,113.02$ & $4,642,513.99$ & Subprimary \\
\hline VG $52 b$ & Valle Guariglia I & Geologic & Recent Neolithic & Maiolica & $584,113.02$ & $4,642,513.99$ & Subprimary \\
\hline VG 52c & Valle Guariglia I & Geologic & Recent Neolithic & Maiolica & $584,113.02$ & $4,642,513.99$ & Subprimary \\
\hline VG 52d & Valle Guariglia I & Geologic & Recent Neolithic & Maiolica & $584,113.02$ & $4,642,513.99$ & Subprimary \\
\hline VG 30_A & Valle Guariglia I & Waste & Recent Neolithic & Maiolica & $584,114.19$ & $4,642,523.50$ & - \\
\hline VG 30_B & Valle Guariglia I & Waste & Recent Neolithic & Maiolica & $584,114.19$ & $4,642,523.50$ & - \\
\hline VG 30_C & Valle Guariglia I & Waste & Recent Neolithic & Maiolica & $584,114.19$ & $4,642,523.50$ & - \\
\hline VG 30_D & Valle Guariglia I & Waste & Recent Neolithic & Maiolica & $584,114.19$ & $4,642,523.50$ & - \\
\hline VG 30_E & Valle Guariglia I & Waste & Recent Neolithic & Maiolica & $584,114.19$ & $4,642,523.50$ & - \\
\hline VG 30_F & Valle Guariglia I & Waste & Recent Neolithic & Maiolica & $584,114.19$ & $4,642,523.50$ & - \\
\hline VG 30_G & Valle Guariglia I & Waste & Recent Neolithic & Maiolica & $584,114.19$ & $4,642,523.50$ & - \\
\hline BRS 50 & Bosco della Risega & Geologic & Neolithic/Copper Age & Maiolica & $587,074.76$ & $4,639,809.27$ & Primary (nodule) \\
\hline BRS 51 & Bosco della Risega & Geologic & Neolithic/Copper Age & Maiolica & $587,074.76$ & $4,639,809.27$ & Primary (nodule) \\
\hline BRS 52 & Bosco della Risega & Geologic & Neolithic/Copper Age & Maiolica & $587,074.76$ & $4,639,809.27$ & Primary (nodule) \\
\hline BRS 53 & Bosco della Risega & Geologic & Neolithic/Copper Age & Maiolica & $587,051.65$ & $4,639,791.71$ & Primary (nodule) \\
\hline BRS 54 & Bosco della Risega & Geologic & Neolithic/Copper Age & Maiolica & $587,051.65$ & $4,639,791.71$ & Primary (nodule) \\
\hline BRS 55 & Bosco della Risega & Geologic & Neolithic/Copper Age & Maiolica & $587,051.65$ & $4,639,791.71$ & Primary (nodule) \\
\hline BRS 56 & Bosco della Risega & Waste & Neolithic/Copper Age & Maiolica & $587,051.65$ & $4,639,791.71$ & Primary (nodule) \\
\hline BRS 57 & Bosco della Risega & Waste & Neolithic/Copper Age & Maiolica & $587,082.46$ & $4,639,757.73$ & Primary (nodule) \\
\hline BRS 58 & Bosco della Risega & Waste & Neolithic/Copper Age & Maiolica & $587,082.46$ & $4,639,757.73$ & Subprimary \\
\hline CRM 10 & Carmine & Geologic & Neolithic/Copper Age & Maiolica & $589,374.37$ & $4,618,972.14$ & Primary (nodule) \\
\hline CRM 11 & Carmine & Geologic & Neolithic/Copper Age & Maiolica & $589,374.37$ & $4,618,972.14$ & Primary (tabular) \\
\hline CRM 12 & Carmine & Geologic & Neolithic/Copper Age & Maiolica & $589,374.37$ & $4,618,972.14$ & Primary (tabular) \\
\hline CRM 13 & Carmine & Geologic & Neolithic/Copper Age & Maiolica & $589,374.37$ & $4,618,972.14$ & Primary (tabular) \\
\hline CRM 14 & Carmine & Geologic & Neolithic/Copper Age & Maiolica & $589,374.37$ & $4,618,972.14$ & Primary (tabular) \\
\hline CRM 15a & Carmine & Geologic & Neolithic/Copper Age & Maiolica & $589,374.37$ & $4,618,972.14$ & Primary (nodule) \\
\hline CRM 16 & Carmine & Geologic & Neolithic/Copper Age & Maiolica & $589,387.06$ & $4,618,941.79$ & Primary (tabular) \\
\hline CRM 17 & Carmine & Geologic & Neolithic/Copper Age & Maiolica & $589,387.06$ & $4,618,941.79$ & Primary (tabular) \\
\hline CRM 18 & Carmine & Geologic & Neolithic/Copper Age & Maiolica & $589,387.06$ & $4,618,941.79$ & Primary (tabular) \\
\hline CRM 19 & Carmine & Geologic & Neolithic/Copper Age & Maiolica & $589,387.06$ & $4,618,941.79$ & Primary (tabular) \\
\hline CRM 20 & Carmine & Geologic & Neolithic/Copper Age & Maiolica & $589,399.97$ & $4,618,898.3$ & Primary (tabular) \\
\hline CRM 21 & Carmine & Geologic & Neolithic/Copper Age & Maiolica & $589,402.01$ & $4,618,881.99$ & Primary (nodule) \\
\hline CRM MIN3B & Carmine & Waste & Neolithic/Copper Age & Maiolica & $589,212.00$ & $4,618,951.00$ & - \\
\hline CRM_30_A & Carmine & Waste & Neolithic/Copper Age & Maiolica & $589,212.00$ & $4,618,951.00$ & - \\
\hline CRM_30_B & Carmine & Waste & Neolithic/Copper Age & Maiolica & $589,212.00$ & $4,618,951.00$ & - \\
\hline CRM_30_C & Carmine & Waste & Neolithic/Copper Age & Maiolica & $589,212.00$ & $4,618,951.00$ & - \\
\hline CRM_30_D & Carmine & Waste & Neolithic/Copper Age & Maiolica & $589,212.00$ & $4,618,951.00$ & - \\
\hline CRM_30_E & Carmine & Waste & Neolithic/Copper Age & Maiolica & $589,212.00$ & $4,618,951.00$ & - \\
\hline CRM_30_F & Carmine & Waste & Neolithic/Copper Age & Maiolica & $589,212.00$ & $4,618,951.00$ & - \\
\hline
\end{tabular}


Table 1 (continued)

\begin{tabular}{|c|c|c|c|c|c|c|c|}
\hline Sample & Mine & Type & Mine chronology & Formation & COOR_E & COOR_N & Position (shape) \\
\hline CRS 50 & Coppa di Rischio & Geologic & Neolithic/Copper Age & Maiolica & $580,871.84$ & $4,640,295.31$ & Primary (nodule) \\
\hline CRS 51 & Coppa di Rischio & Geologic & Neolithic/Copper Age & Maiolica & $580,871.84$ & $4,640,295.31$ & Primary (nodule) \\
\hline CRS 52 & Coppa di Rischio & Geologic & Neolithic/Copper Age & Maiolica & $580,871.84$ & $4,640,295.31$ & Primary (nodule) \\
\hline CRS 53 & Coppa di Rischio & Geologic & Neolithic/Copper Age & Maiolica & $580,871.84$ & $4,640,295.31$ & Primary (nodule) \\
\hline CRS 54 & Coppa di Rischio & Geologic & Neolithic/Copper Age & Maiolica & $580,866.86$ & $4,640,274.24$ & Subprimary \\
\hline CRS 55 & Coppa di Rischio & Geologic & Neolithic/Copper Age & Maiolica & $580,866.86$ & $4,640,274.24$ & Primary (nodule) \\
\hline MST T1 & Mastro Tonno & Waste & Neolithic/Copper Age & Maiolica & $584,369.00$ & $4,641,625.00$ & - \\
\hline MST T2 & Mastro Tonno & Waste & Neolithic/Copper Age & Maiolica & $584,369.00$ & $4,641,625.00$ & - \\
\hline MST 30_A & Mastro Tonno & Waste & Neolithic/Copper Age & Maiolica & $584,369.00$ & $4,641,625.00$ & - \\
\hline MST 30_B & Mastro Tonno & Waste & Neolithic/Copper Age & Maiolica & $584,369.00$ & $4,641,625.00$ & - \\
\hline MST 30_C & Mastro Tonno & Waste & Neolithic/Copper Age & Maiolica & $584,369.00$ & $4,641,625.00$ & - \\
\hline MST 30_E & Mastro Tonno & Waste & Neolithic/Copper Age & Maiolica & $584,369.00$ & $4,641,625.00$ & - \\
\hline MST 30_F & Mastro Tonno & Waste & Neolithic/Copper Age & Maiolica & $584,369.00$ & $4,641,625.00$ & - \\
\hline MST 30_G & Mastro Tonno & Waste & Neolithic/Copper Age & Maiolica & $584,369.00$ & $4,641,625.00$ & - \\
\hline MST 30_H & Mastro Tonno & Waste & Neolithic/Copper Age & Maiolica & $584,369.00$ & $4,641,625.00$ & - \\
\hline CR1a & Cruci & Geologic & Copper Age & Maiolica & $582,855.99$ & $4,641,578.98$ & - \\
\hline CR1b & Cruci & Geologic & Copper Age & Maiolica & $582,855.99$ & $4,641,578.98$ & - \\
\hline CR1c & Cruci & Geologic & Copper Age & Maiolica & $582,855.99$ & $4,641,578.98$ & - \\
\hline CR50 & Cruci & Geologic & Copper Age & Maiolica & $582,855.99$ & $4,641,578.98$ & Primary (nodule) \\
\hline CR51 & Cruci & Geologic & Copper Age & Maiolica & $582,855.99$ & $4,641,578.98$ & Primary (nodule) \\
\hline CR52 & Cruci & Geologic & Copper Age & Maiolica & $582,801.06$ & ,4,641,601.09 & Primary (nodule) \\
\hline CR53 & Cruci & Geologic & Copper Age & Maiolica & $582,802.06$ & ,4,641,601.10 & Primary (nodule) \\
\hline CR54 & Cruci & Geologic & Copper Age & Maiolica & $582,773.19$ & $4,641,599.96$ & Primary (nodule) \\
\hline MRT1a & Martinetti & Geologic & Copper Age & Maiolica & $583,584.89$ & $4,641,164.55$ & - \\
\hline MRT1b & Martinetti & Geologic & Copper Age & Maiolica & $583,584.89$ & $4,641,164.55$ & - \\
\hline MRT1c & Martinetti & Geologic & Copper Age & Maiolica & $583,584.89$ & $4,641,164.55$ & - \\
\hline MRT1up & Martinetti & Geologic & Copper Age & Maiolica & $583,584.89$ & $4,641,164.55$ & - \\
\hline MRT50 & Martinetti & Geologic & Copper Age & Maiolica & $583,584.89$ & $4,641,164.55$ & Primary (nodule) \\
\hline MRT51 & Martinetti & Geologic & Copper Age & Maiolica & $583,584.89$ & $4,641,164.55$ & Primary (nodule) \\
\hline MRT52 & Martinetti & Geologic & Copper Age & Maiolica & $583,584.89$ & $4,641,164.55$ & Primary (nodule) \\
\hline MRT53 & Martinetti & Geologic & Copper Age & Maiolica & $583,584.89$ & $4,641,164.55$ & Primary (nodule) \\
\hline MRT54 & Martinetti & Geologic & Copper Age & Maiolica & $583,584.89$ & $4,641,164.55$ & Primary (nodule) \\
\hline TGC3 & Tagliacantoni & Waste & Copper Age & Scaglia & $587,234.92$ & $4,642,669.91$ & - \\
\hline TGC4 & Tagliacantoni & Waste & Copper Age & Scaglia & $587,234.92$ & $4,642,669.91$ & - \\
\hline TGC50 & Tagliacantoni & Geologic & Copper Age & Scaglia & $587,342.76$ & $4,642,524.93$ & Primary (tabular) \\
\hline TGC51 & Tagliacantoni & Geologic & Copper Age & Scaglia & $587,342.76$ & $4,642,524.93$ & Primary (tabular) \\
\hline TGC52 & Tagliacantoni & Geologic & Copper Age & Scaglia & $587,342.76$ & $4,642,524.93$ & Primary (tabular) \\
\hline TGC53 & Tagliacantoni & Geologic & Copper Age & Scaglia & $587,223.14$ & $4,642,693.47$ & Primary (nodule) \\
\hline TGC54a & Tagliacantoni & Geologic & Copper Age & Scaglia & $587,223.14$ & $4,642,693.47$ & Subprimary \\
\hline TGC 54b & Tagliacantoni & Geologic & Copper Age & Scaglia & $587,223.14$ & $4,642,693.47$ & Subprimary \\
\hline TGC $54 \mathrm{c}$ & Tagliacantoni & Geologic & Copper Age & Scaglia & $587,223.14$ & $4,642,693.47$ & Subprimary \\
\hline TGC 54d & Tagliacantoni & Geologic & Copper Age & Scaglia & $587,223.14$ & $4,642,693.47$ & Subprimary \\
\hline TGC $54 \mathrm{e}$ & Tagliacantoni & Geologic & Copper Age & Scaglia & $587,223.14$ & $4,642,693.47$ & Subprimary \\
\hline TGC 54f & Tagliacantoni & Geologic & Copper Age & Scaglia & $587,223.14$ & $4,642,693.47$ & Subprimary \\
\hline TGC $54 \mathrm{~g}$ & Tagliacantoni & Geologic & Copper Age & Scaglia & $587,223.14$ & $4,642,693.47$ & Subprimary \\
\hline TGC 54h & Tagliacantoni & Geologic & Copper Age & Scaglia & $587,223.14$ & $4,642,693.47$ & Subprimary \\
\hline TGC $54 \mathrm{i}$ & Tagliacantoni & Geologic & Copper Age & Scaglia & $587,223.14$ & $4,642,693.47$ & Subprimary \\
\hline TGC 541 & Tagliacantoni & Geologic & Copper Age & Scaglia & $587,223.14$ & $4,642,693.47$ & Subprimary \\
\hline TGC $54 \mathrm{~m}$ & Tagliacantoni & Geologic & Copper Age & Scaglia & $587,223.14$ & $4,642,693.47$ & Subprimary \\
\hline
\end{tabular}


Table 1 (continued)

\begin{tabular}{llllllll}
\hline Sample & Mine & Type & Mine chronology & Formation & COOR_E & COOR_N & Position (shape) \\
\hline GR-SB_a & Valle Sbernia/Guariglia & Geologic & Copper Age & Maiolica & $584,127.98$ & $4,642,042.96$ & - \\
GR-SB_b & Valle Sbernia/Guariglia & Geologic & Copper Age & Maiolica & $584,127.98$ & $4,642,042.96$ & - \\
GR-SB_c & Valle Sbernia/Guariglia & Geologic & Copper Age & Maiolica & $584,127.98$ & $4,642,042.96$ & - \\
GRSB50 & Valle Sbernia/Guariglia & Geologic & Copper Age & Maiolica & $584,129.95$ & $4,642,051.23$ & Primary (nodule) \\
GRSB52 & Valle Sbernia/Guariglia & Geologic & Copper Age & Maiolica & $584,129.95$ & $4,642,051.23$ & Subprimary \\
GRSB54 & Valle Sbernia/Guariglia & Geologic & Copper Age & Maiolica & $584,131.76$ & $4,642,022.91$ & Primary (tabular) \\
GRSB55 & Valle Sbernia/Guariglia & Geologic & Copper Age & Maiolica & $584,131.76$ & $4,642,022.91$ & Primary (tabular) \\
GRSB56 & Valle Sbernia/Guariglia & Geologic & Copper Age & Maiolica & $584,134.94$ & $4,642,005.01$ & Primary (nodule) \\
\hline
\end{tabular}

\section{Mine typology and extractive districts}

Isolated mining sites and mining complexes have been identified in the Gargano area; Carmine, Bosco della Risega, Tagliacantoni and Coppa di Rischio belong to the former group, consisting of a sequence of adjacent mining facilities. Mining complexes are instead composed of groups of mining sites excavated in a specific area marked by homogeneous geomorphology and topography (Tarantini and Galiberti 2011). The mining complex of Vieste is located near the town of Vieste close to the coastline and covers a surface area of approximately $4 \mathrm{~km}^{2}$. Evidence of mining investigated in this work has been identified in the hills of Intraseglio, where Defensola $\mathrm{A}$ is located, as well as the hills of Defensola, where Defensola C, Defensola B/P9, Defensola B/P8 and San Marco, with the mine of the same name, are located.

Holocene paleoenvironmental changes in the lower valley at the bottom of the hill have been detected (Caroli and Caldara 2007; Caldara et al. 2008). The Peschici Fm. shows medium- and large-sized nodules in the pelagic facies positioned in the lower part and decreasing at the top, where resedimented deposits prevail. Mining structures are positioned at the bottom of sequences where the Peschici Fm. unconformably overlies the Scaglia Fm., causing some stratigraphic uncertainties, as in the case of Tagliacantoni (Tarantini and Galiberti 2011). The mining complex of Peschici is located in the Peschici hinterland. Mining sites are positioned in the hills facing the Ulso valley, where the mines of Cruci, Valle Sbernia/ Guariglia, Valle Guariglia I and Mastro Tonno have been identified, while Martinetti is situated in a flat area at approximatelyan elevation of approximately $100 \mathrm{~m}$ altitude (Tarantini and Galiberti 2011).

Three main typologies of chert extraction have been indicated in the investigations by Tarantini and Galiberti (2011), denominated as type 'A', 'B' and 'C'. Type A is observed in compact limestone and has developed subhorizontal mining extraction described as rooms and pillars'. The mines Defensola A, Defensola C, San
Marco and Martinetti belong to subtype A1, characterized by only horizontal entrances. The Arciprete mine represents subtype A2, with a combination of horizontal entrances and shaft mine entrances. Variations are observed in Tagliacantoni (subtype A1b) and Defensola B (subtype A1a) mines. Mining structures of type B are characterized by exclusively vertical shaft entrances, and three subtypes have been discriminated: $(i)$ subtype B1, with a vertical or slightly sloping shaft, as in the cases of Martinetti, Valle Guariglia I and Carmine; (ii) subtype B2, with a vertical shaft ending in a large excavation, as in the cases of Coppa di Rischio and Martinetti; and (iii) subtype B3, with several shafts ending in a connected subhorizontal excavation, as in the cases of Valle Sbernia/Guariglia and Bosco della Risega.

Mining structures of type $\mathrm{C}$ are characterized by many vertical shafts, and later horizontal entrances are visible in Cruci, Martinetti, Mastro Tonno, Defensola B/P9, Coppa di Rischio and Tagliacantoni mines; frequently, the shape of the horizontal entrance is similar to a dromos.

\section{Sampling strategy}

The sampling strategy has developed in two stages. The first stage is related to the geological study of the territory, resulting in a source map of the Gargano Promontory, as described above. The second stage is related to field sampling. The geologic map of the Gargano area (Fig. 1) includes only the geological formations effectively or potentially exploited by prehistoric communities. Primary potential sources such as the Marne a Fucoidi, Monte S. Angelo and Monte Acuto Fms. reveal no evidence of mining, whereas secondary sources have been identified among coastal and continental Quaternary deposits.

Chert samples were collected mainly from nodules in primary position and frequently from those in subprimary positions. Mines intersected by the road or near outcrops of cherty levels allowed sampling in a systematic way in order to check lateral and vertical homogeneity of the 
chert-bearing formations, as in the cases of the Carmine, Tagliacantoni and several Maiolica mines.

NM-PCI was applied to a total of 155 Gargano samples collected during the field sampling from 15 mine sites in the three mining districts of Peschici, Vieste and Mattinata. Geological and archaeological information about each sample is reported in Table 1.

For the mines of Arciprete and Mastro Tonno, only archaeological waste samples (Tarantini and Galiberti 2011) were analysed, given the inaccessibility of the sites. Patinated samples were excluded from the analysis. A selection of examined materials is shown in Figs. 3 and 4.

\section{Multiparametric method (NM-PCI)}

The proposed method aimed to obtain macro/microscopic and geochemical data using exclusively nondestructive procedures (Fig. 5).

In each analytical step, data of different types were collected through a formalized protocol to yield a mixed data matrix of specific characteristics of chert: structure, colour and principal structures, texture and chemical composition. This strategy of data collection was functional for the subsequent multivariate statistical treatment.

Archaeological approaches commonly compare the visual properties of chert artifacts and raw materials (mainly local sources) in order to correlate them. Subjective descriptions of samples occur for the same visible chert characteristics. These characteristics can often be observed from various geological units and different geographic areas. The identification of geological sources for chert artifacts is then problematic when using only visual identification, since the variation in physical properties can be large, sometimes even within a single nodule (Luedtke 1979; Selivanova 1986). Moreover, the use of geochemistry for chert sourcing is less distinctive than for obsidian sourcing due to petrological reasons. Instead, the simultaneous use of a number of (semi)quantitative variables, processed with multivariate statistics, may be more effective in provenance and techno-functional studies.

To gain the maximum information from samples, it is generally important to work on clean fresh surfaces. Since weathered and patinated surfaces may obliterate visible structures of chert and alter chemical composition, only unaltered samples were considered for the analyses.

\section{Data collection}

\section{Macroscopic description}

Prehistoric stoneworkers gained experience observing the visible properties of knappable rocks. The selection of raw materials was in part guided by the ability to distinguish between good and poor quality raw materials. The simultaneous study of geological and archaeological samples allows researchers to understand which desired traits recur and which visible properties may have mainly influenced the choice. Since chert generally shows a widely variable appearance, examination with the naked eye is fundamental to recognising its typical characteristics but can possibly lead to errors because of subjective descriptions and inter-observer errors. To evaluate the frequency of each feature and to understand potential existing nonaccidental correlations between the variables, it is

Fig. 3 Samples from the mines of the Peschici district. Bosco della Risega (1 and 2), Valle Sbernia/ Guariglia (3), Martinetti (4), Coppa di Rischio (5 and 6), Valle Guariglia I (7 and 12),

Tagliacantoni (8 and 9), Cruci (10 and 11) and Mastro Tonno (13). Sample 13 was photographed at a different scale

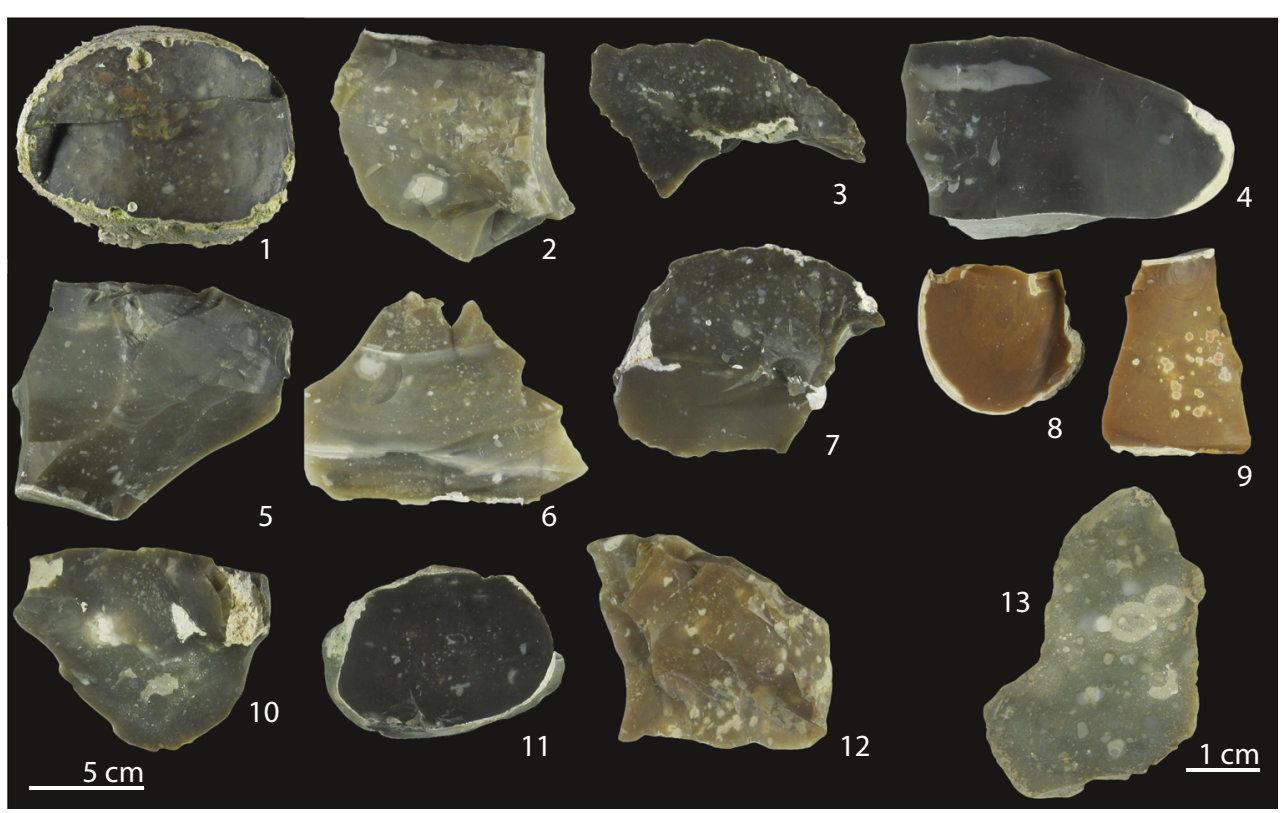


Fig. 4 Samples from the mines of the Vieste and Mattinata districts. Defensola B/P8 (1 and 2), Defensola C (3 and 4), Carmine (5 and 6), San Marco (7), Defensola B/P9 (8 and 9), Defensola A (10 and 11) and Arciprete (12 and 13). Samples $10,11,12$ and 13 were photographed at a different scale

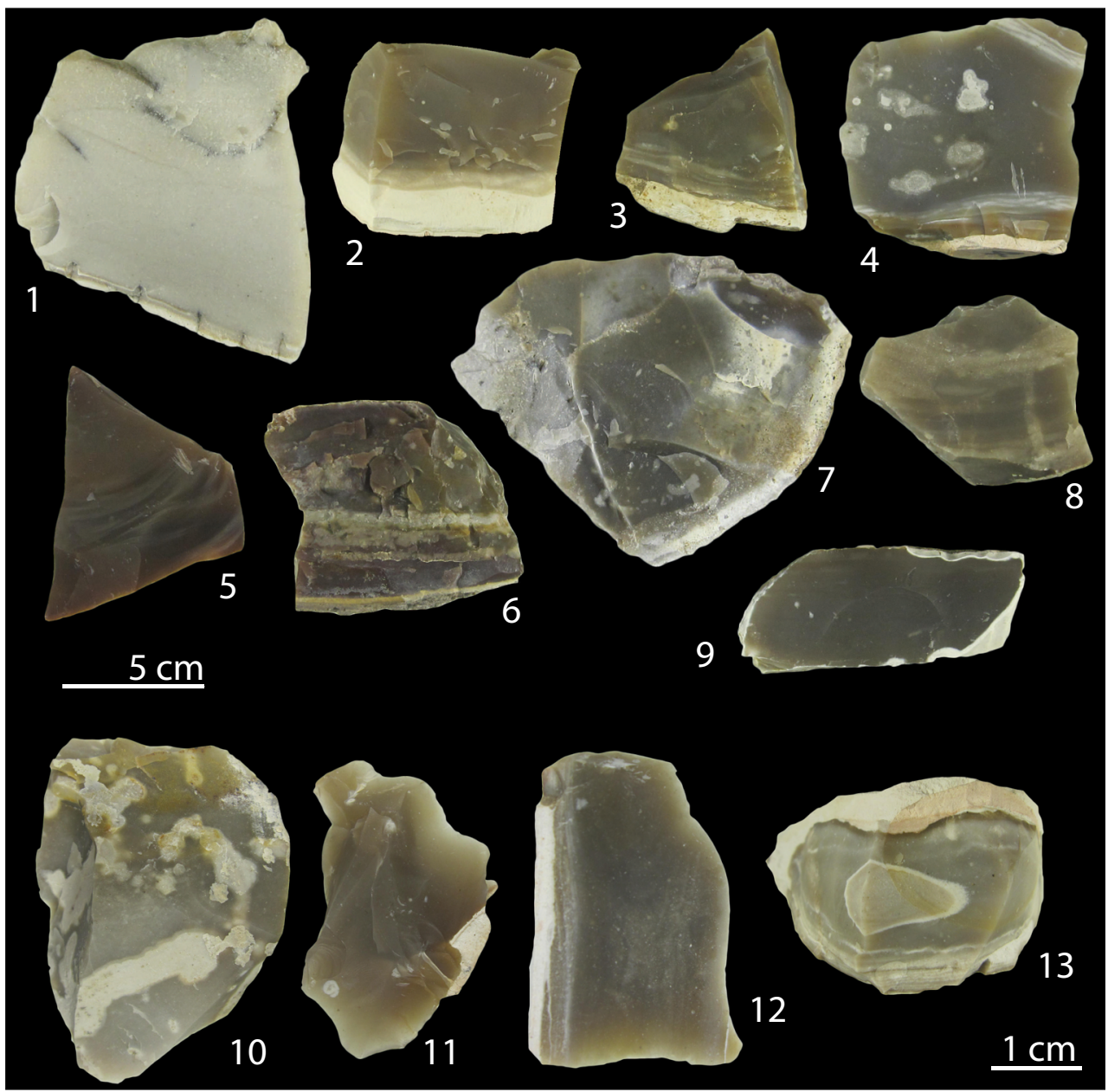

necessary to follow a standardized protocol of measurement at different scales to isolate and objectively document the sample. The method proposed here is based on the multiparametric protocol developed by Tarantini et al. (2016) and implemented with new microscopic observations to better describe microfacies and micropaleontological features. The mixed data matrix is structured in two parts that describe the cortex and the interior (s.s.) portions of the chert on macroscopic and microscopic scales. When present, the cortex constitutes the outer portion, which is frequently visible in many chert types. During the process of silicification, the cortex represents the transition zone between the chert and the surrounding bedrock and is characterized by a distinct colour and chemical composition (Luedtke 1992). The cortex zone is described by five macroscopic parameters: thickness, nature, induration, surface and boundary. Since all analysed cherts were primary, information about the neocortex and secondary neocortex (Fernandes et al. 2007; Fernandes and Raynal 2010; Fernandes 2012) is not considered in the description. The maximum thickness of the visible cortex is measured with a caliper and expressed in millimetres. The chemical nature of the cortex is determined by means of an acid test. A drop of $2 \% \mathrm{HCl}$ solution deposited on the fresh cortex surface discriminates between its carbonate and siliceous nature. The induration is estimated with a steel penknife. If the fresh cortex surface is scratched, it is classified as friable; otherwise, it is hard. Three terms describe the outer surface of the cortex: harsh, rough or smooth to the touch. Finally, the boundary, which represents the visible limit of distinction between chert and cortex, is described as sharp, clear or diffuse, based on the clarity of the boundary. A sharp boundary is characterized by an abrupt change in colour and texture that is visible between the cortex and the chert domain. In contrast, a faint change in colour and texture distinguishes a diffuse boundary, whereas a clear boundary is an intermediate condition. The macroscopic description proceeds with chert observations of the subcortex, structure and fracture. The subcortex, when present, is the transition zone between the cortex and the chert. Seven basic categories are identified for chert structure(s): homogeneous, shaded, mottled, spotted, laminated, banded and streaked. This variable regards the overall appearance of the rock, including the colour distribution, and may consider more than one basic structure. The observer must distinguish between matrix 
Fig. 5 Flow chart of the NM-PCI method

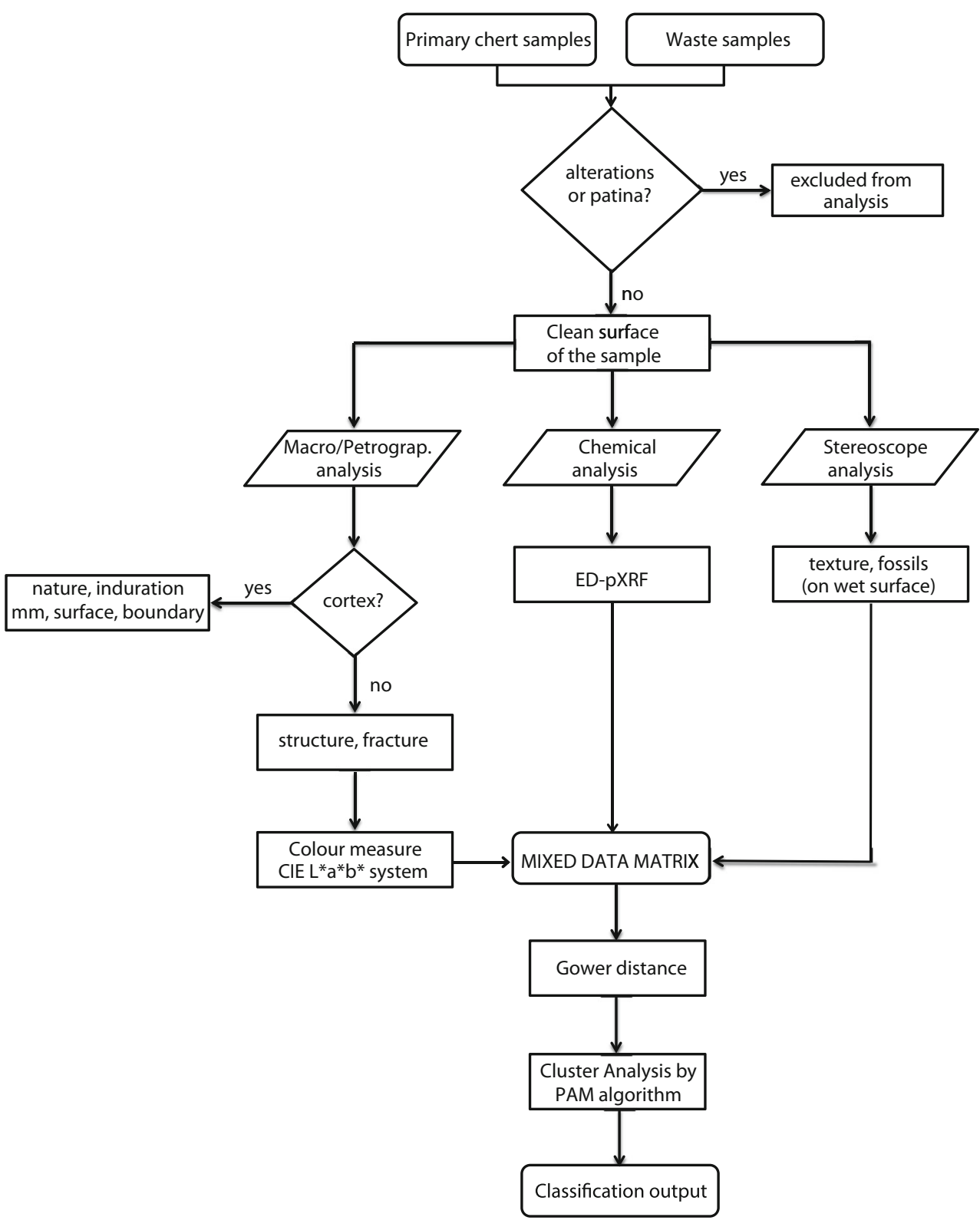

types and biogenic or inorganic grains $(\varnothing \geq 63 \mu \mathrm{m}$, after Dunham 1962), visible structures such as bedding and lamination and a range of possible deformation of the primary structures caused by postdepositional processes. A homogeneous structure corresponds to a chert with a uniform appearance that contains an amount of grains $<10$ vol. $\%$. The remaining six categories can document, singularly or in combination, all the possible degrees of heterogeneity. Small spots of light colour, distributed within the matrix and with sizes between 0.63 and $4 \mathrm{~mm}$, identify a spotted structure. Mottled is a structure characterized by pale blotchy, marbled, uneven inclusions larger than $4 \mathrm{~mm}$. When inhomogeneity occurs in the colour of the matrix, exhibiting gradual transitions, chert can be defined as shaded. Based on the thickness of visible layers, the structure is identified as laminated if the layer thickness is less than $1 \mathrm{~cm}$ and banded if the thickness is greater than $1 \mathrm{~cm}$. Finally, streaked indicates a structure with stretched, lighter and marbled heterogeneities.

The last variable that completes the macroscopic description is the fracture, a physical property of minerals based on the strength of the crystal structure bonds (Klein et al. 1993) and observable in hand specimens, which describes the way minerals break when subject to mechanical stresses. Since chert is a siliceous rock, the quality of fracture is also a function of texture, structure and porosity (vugs and/or fissures). Fracture patterns can be identified as conchoidal, marked by smooth and curved surfaces with visible ripples, to subconchoidal, lacking ripples and irregular or uneven. 


\section{Mesoscopic description}

After the description with the naked eye, the observations continue at low magnification on wet surfaces of the samples by means of a stereomicroscope, according to the following standardized procedure. A thin film of water ensures a smoother surface by increasing light reflection from the upper surface, thus improving the optical conditions. The chert is classified as translucent or opaque according to whether inclusions are distinguished or not, below the illuminated surface at $\times 10$ magnification.

The surfaces of each sample are accurately examined and matched with comparison charts (Matthew et al. 1991) to estimate the percentage of allochems and their size range.

Thus, a sample may be well, moderately or poorly sorted and with three possible amounts of particles. Sometimes, samples exhibit two different sorting or percentage classifications. In these cases, all the data are duplicated, and observations are split into two rows. At $\times 40$ magnification, the preserved fossil assemblages are identified (e.g. foraminifera, radiolarians and sponge spicules). A semiquantitative scale ( $0-3)$ is used to indicate the fossil abundance, where 0 indicates absence, 1 indicates a number of organisms equal to or less than three (traces), 2 indicates minor amounts (number of organisms between four and ten) and 3 indicates major amounts (number of organisms higher than ten).

\section{Colour}

The measurement of the colour(s) of the chert is performed with a noninvasive spectrophotometric technique. To obtain reproducible colour measurements as continuous variables easy to process statistically, the protocol recommends acquiring colour coordinates in the Commission Internationale de l'Eclairage (CIE) $L^{*} a^{*} b^{*}$ colour system (McLaren 1976) from only the matrix portion, as it is more homogeneous. The three coordinates are $L^{*}$, lightness $(0-100) ; a^{*}$, red/green value $( \pm 100)$; and $b^{*}$, yellow/blue value $( \pm 100)$. A portable spectrophotometer, Konica Minolta CM-2600d, is employed to acquire colour data in the CIE $L^{*} a^{*} b^{*}$ colour system in the wavelength range from 360 to $740 \mathrm{~nm}$ with a wavelength step size of $10 \mathrm{~nm}$. Color coordinates result from processing the measured reflectance spectra (Konica Minolta instruction manual). The colorimetric information provided for the NM-PCI is represented by the three values of $L^{*}, a^{*}$ and $b^{*}$, which avoids excess data.

The instrumental settings employed for the spectrophotometer were as follows: an aperture mask with a diameter of $6 \mathrm{~mm}$ in, appropriate to isolate small homogeneous portions of matrix on the sample, a D65 illuminant (natural daylight) and a standard observer at a $10^{\circ}$ angle. Both specular component included (SCI) and specular component excluded (SCE) were acquired. UV radiation is excluded to avoid interference with colour measurement due to fluorescence phenomena
(Berger-Schunn 1994). Moreover, a regular calibration of the instrument was performed with a white standard and with a black body, in order to obtain $100 \%$ and $0 \%$ lines of reflectance. The sample surface was cleaned with a $2 \% \mathrm{HCl}$ water solution before colour acquisition, and each measurement was replicated three times in the same position of the chert and averaged. Sometimes the samples show two or more hues distinguishable by the naked eye. In this case, two or more series of colour measurements were carried out. When a sample had a complex geometry, it was not always possible to take colour measurements.

\section{Geochemistry}

Elemental analysis was carried out using a portable ThermoNITON XL3t XRF spectrometer. The device was equipped with a Ag collimated source operating at a maximum of $2 \mathrm{~W}$ and an SDD detector. The spot size was approximately $3 \mathrm{~mm}$ in diameter, and the resolution of the detector was lower than $160 \mathrm{eV}$. To quantify both light and heavy elements, a total of $120 \mathrm{~s}$ of realtime acquisition was used. Specifically, the sample was analysed for $60 \mathrm{~s}$ at $40 \mathrm{kV}$ and $50 \mu \mathrm{A}$, for $30 \mathrm{~s}$ at $50 \mathrm{kV}$ and $40 \mu \mathrm{A}$ and finally for $30 \mathrm{~s}$ at $20 \mathrm{kV}$ and $100 \mu \mathrm{A}$. At least two measures on the matrix of each sample were acquired. The concentrations of eight elements ( $\mathrm{Fe}, \mathrm{K}, \mathrm{Sr}, \mathrm{Rb}, \mathrm{Ni}, \mathrm{Mn}, \mathrm{As}$ and $\mathrm{Ba}$ ) were determined. These elements presented only a few values below the limit of detection (LOD) and a good accuracy of measurement compared to the standards (TILL- 4 by Canadian Certified Reference Material Project), as shown in Table 2. The LOD was determined as three times the standard deviation of the replicated blank signal ( $3 \sigma$ method). All samples presenting a clear surface patina easily visible to the naked eye were excluded from chemical analysis. The XRF spectrometer was calibrated using a set of geological Service of Analyzes des Roches et des Minéraux (SARM, CRPG-CNRS, Vandoeuvre-les-Nancy, France) standards, including those in particular silicate rocks.

Table 2 pXRF analysis of TILL-4 reference material and the limit of detection (LOD) of the selected elements

\begin{tabular}{|c|c|c|c|c|c|}
\hline \multirow[b]{2}{*}{ Element } & \multicolumn{2}{|l|}{ Certificate } & \multicolumn{3}{|l|}{$\mathrm{pED}-\mathrm{XRF}$} \\
\hline & Concentration & Std. Dev. & Concentration & Std. Dev. & LOD \\
\hline K & $2.7(\%)$ & 0.1 & $2.73(\%)$ & $0.01(\%)$ & 50 \\
\hline $\mathrm{Fe}$ & $3.97(\%)$ & 0.15 & $3.47(\%)$ & $0.01(\%)$ & 8 \\
\hline As & 111 & 6 & 113 & 1 & 1 \\
\hline $\mathrm{Ba}$ & 395 & 37 & 407 & 4 & 68 \\
\hline $\mathrm{Mn}$ & 490 & 77 & 441 & 6 & 10 \\
\hline $\mathrm{Ni}$ & 17 & 3 & 20 & 5 & 10 \\
\hline $\mathrm{Rb}$ & 161 & 15 & 149 & 3 & 1 \\
\hline $\mathrm{Sr}$ & 109 & 11 & 118 & 1 & 1 \\
\hline
\end{tabular}

The concentrations are expressed in milligrams per kilogram, except for $\mathrm{K}$ and $\mathrm{Fe}$ 


\section{Data processing}

\section{Transformation}

Following the data collection phase, a set of cases in a mixed data sheet was created. Cluster analysis (CA) is a technique of exploratory analysis that is very common in the case of unsupervised learning for examining unstructured multivariate data (Izenman 2008) and is frequently applied in archaeometric investigations (Baxter 1994). The purpose of clustering is to identify groups of observations by means of the measurement of proximity (dissimilarity distance or similarity between each pair of observations) in such a way that statistical units (i.e. cases) group into a cluster sharing a series of common features (Everitt et al. 2011). Generally, a data matrix includes a large number of cases greater than the number of variables, and the goal of multivariate statistics is to segment the starting data set into small, homogeneous subgroups or clusters of cases. To perform a clustering procedure, it is necessary to choose the appropriate proximity measure and the group-building algorithm in order to assign each case to a cluster so that the similarities between the cases of the same cluster are maximized and those between clusters are minimized (James et al. 2013). The software environment used to perform data processing was $\mathrm{R}$ ( $\mathrm{R}$ Core Team 2018), a freeware package for statistical computing and graphics. The mixed data matrix obtained from NM-PCI consisted of categorical (e.g. texture, structure), ordinal (fracture, micropaleontological content) and continuous variables (colour coordinates, elemental concentrations). To statistically process the categorical variables, they were transformed into binary variables, assigning the value 0 or 1 to each category according to its absence or presence in the sample. The most suitable clustering algorithm was chosen based on mixed datasets. Hierarchical clustering and $K$-means were excluded because they do not handle heterogeneous data or dissimilarity matrices. A similarity measure for mixed-mode data can be obtained by Gower's similarity coefficient:

$$
S i j=\frac{\sum_{k=1}^{p} S_{i j k} \delta_{i j k}}{\sum_{k=1}^{p} \delta_{i j k}}
$$

where $s_{i j}$ expresses the similarity between two individuals $i$ and $j$ relative to a given $k^{\text {th }}$ variable; the quantity $\delta_{i j k}$ assumes a value equal to 1 when the comparison is accepted as valid and otherwise is set to 0 . This index corresponds to the weighted average of the distances calculated for each variable and returns a number in the range between 0 and 1 expressing identity or complete dissimilarity, respectively. The complement of the similarity measure is the dissimilarity measure (Gower 1971).

In the case of binary and categorical variables, $s_{i j k}$ equals 1 when the same value is observed for the two individuals and equals 0 otherwise. If both variables are continuous, Gower (1971) suggested using the similarity measure: $s_{i j}=1-x_{i k}-$ $x_{j k} / R_{k}$, where $R_{k}$ corresponds to the range of entries for the $k^{\text {th }}$ variable (Everitt et al. 2011).

\section{Multivariate statistics}

The clustering algorithm chosen was the partitioning around medoids or PAM (Kaufman and Rousseeuw 1990), a revised form of the K-medoids algorithm (MacQueen 1967), which is also able to process dissimilarity matrices. This algorithm is an iterative clustering practice that detects objects called medoids to build clusters. A medoid constitutes the most centrally positioned object in a cluster, in contrast to centroids used in $K$ means clustering (MacQueen 1967). Since PAM minimizes the sum of dissimilarities instead of the sum of squared Euclidean distances, it is considered a 'robust' method to treat anomalous data (i.e. outliers and missing values) (Izenman 2008; Borcard et al. 2011). Given the proximity matrix and an initial setup of $k$ number of clusters, the algorithm works according to a building and then swapping strategy, which first randomly selects $k$ in the data set and assigns each case to the nearest medoid; then, by iterative searches, the medoid of each cluster is substituted by another member of the cluster if the new nonmedoid configuration minimizes the total dissimilarity of the cluster. The process is repeated until no changes in the medoids occur (Reynolds et al. 2006; Izenman 2008; Bhat 2014).

\section{Validation}

Although the number of $k$ clusters required for clustering was fixed in this work (15, according to the number of the studied mines), the silhouette function is used to evaluate the outcome of PAM on the data set. The clustering structure obtained after the partition process can be evaluated by means of a silhouette plot, which provides useful guidance to assess the quality of clusters, detects the misclassified cases and identifies the appropriate number of clusters for the data set under investigation (Rousseeuw 1987). The partition result $C_{k}$ distributes the cases into $k$ clusters; for each $i^{\text {th }}$ entry in the cluster, $a_{i}$ is calculated, which is the average dissimilarity of the $i^{\text {th }}$ case to all other cases belonging to the same cluster. The cluster of belonging is excluded from calculations, and the average dissimilarity of the $i^{\text {th }}$ case from all cases to all other clusters $C$ is also calculated as $d(i, C)$; the smallest dissimilarity $b_{i}=\min _{c \text { - }}$ $d(i, C)$ represents the neighbor cluster of the $i$ th case. The silhouette width of the $i_{\mathrm{th}}$ entry is equal to $s_{i}=b_{i}-a_{i} / \max \{a$ $\left.{ }_{i}, b_{i}\right\}$ and ranges from -1 to 1 . When $S_{i}$ is positive and close to 1 , the object is well clustered. Conversely, when $S_{i}$ is negative, the case is probably misclassified in the wrong cluster. When $S_{i}$ is almost zero, it indicates that the $i^{\text {th }}$ case lies between two clusters (Kaufman and Rousseeuw 1990; Izenman 2008). In 
the silhouette plot, all $S_{i}$ for each cluster are ranked in decreasing order and shown with a bar plot. Furthermore, the average silhouette width of the entire clustering structure is displayed on the plot. This value is a suitable indicator of clustering result $C_{k}$ and can also be used to choose the value $k$ clusters, while the silhouette coefficient $S C=\max _{k}\left\{\delta_{k}\right\}$ helps to interpret the clustering structure (Kaufman and Rousseeuw 1990). The silhouette width is usually used to assess the goodness of clustering for internal clustering evaluation (Kassambara 2017), as the Dunn index (Dunn 1974), which defines the ratio between the minimal intracluster distance and the maximal intercluster distance; this value ranges in the interval and should be maximized to improve the goodness of clustering.

To compare the partitioned structure to a correct classification, external clustering validation practices are useful indicators to evaluate case classification, such as the Rand index (Rand 1971), which ranges from -1 (misclassification) to 1 (perfect agreement).

Given the large number of variables, the outcome must be visualized, and a very useful tool for dimensionality reduction is the $t$-distributed stochastic neighbor embedding (t-SNE). Through this method, it is possible to display the clusters in a two- or three-dimensional map, preserving local structures (Maaten and Hinton 2008).

When a plateau region occurs in the silhouette plot, the Dunn and Rand indices are compared to select the most suitable $k$ value.

Preprocessing of geochemical data ensures that data below the LOD are replaced with a new value close to the LOD, which is approximately one-tenth of the lowest value measured for the variable. The script was run using $\mathrm{R}$ version 3.4.3. Four packages were used for data processing: cluster (Maechler et al. 2018) for the Gower similarity measure, PAM algorithm and silhouette width; ggplot2 (Wickham 2016) and Rtsne (Krijthe 2015) for result visualisation; and fpc (Hennig 2014) for internal and external clustering validation. After loading the matrix in $\mathrm{R}$, in order to build a statistical model based on heterogeneous data type, it is necessary to turn categorical variables into factors (each with specific factor levels). At this point, the column of labels is removed from the matrix, and dissimilarity measures are calculated by setting the metric equal to 'Gower' in the daisy function.

\section{Results}

\section{Petrography}

\section{Cortex}

The outer layer is not always observable using archaeological tools, and thus, the features that characterize it are excluded from the statistical procedure in the provenance study. Geological or waste samples with a visible cortex in the study are 117, and the data are summarized for each mine in Table 3.

The mean thickness and the more frequent features per variable are presented in Table 3, separated with a slash when two features are equally frequent.

In summary, the data in Table 3 clearly show that the cortexes of the cherts from the Arciprete, Defensola A and San Marco mines are very similar, while those from the other

Table 3 More frequent features of the cortex identified in the chert from the Gargano mines

\begin{tabular}{|c|c|c|c|c|c|c|c|}
\hline Mine & Samples $(n)$ & Mean thickness (mm) & Nature & Induration & Surface & Boundary & Subcortex \\
\hline Arciprete & 8 & 3 & Siliceous & Hard & Smooth & Sharp & Present $(50 \%)$ \\
\hline Defensola A & 5 & 4 & Siliceous & Hard & Smooth & Sharp & Absent \\
\hline Defensola B/P9 & 11 & 2 & Siliceous/calcareous & Hard/friable & Smooth/rough & Clear & Present $(63 \%)$ \\
\hline Defensola B/P8 & 8 & 6 & Calcareous & Hard/friable & Rough & Sharp/diffuse & Present $(50 \%)$ \\
\hline Defensola C & 6 & 7 & Calcareous & Friable & Rough & Clear/sharp & Present $(66 \%)$ \\
\hline San Marco & 7 & 5 & Siliceous & Hard & Smooth & Diffuse/clear/sharp & Present $(57 \%)$ \\
\hline Bosco della Risega & 9 & 4 & Calcareous & Friable & Rough & Sharp & Present $(11 \%)$ \\
\hline Carmine & 14 & 4 & Calcareous & Friable & Rough & Sharp & Present (14\%) \\
\hline Coppa di Rischio & 6 & 3 & Calcareous & Friable & Rough & Sharp & Present $(17 \%)$ \\
\hline Cruci & 4 & 2 & Calcareous & Friable & Rough & Sharp & Present $(25 \%)$ \\
\hline Martinetti & 3 & 6 & Siliceous/calcareous & Friable & Hard/rough & Sharp & Absent \\
\hline Mastro Tonno & 7 & 2 & Calcareous & friable & rough & sharp & Absent \\
\hline Valle Guariglia I & 9 & 1 & Calcareous & friable & rough & sharp & Present $(11 \%)$ \\
\hline Valle Sbernia/Guariglia & 8 & 3 & Calcareous & friable & rough & sharp & Absent \\
\hline Tagliacantoni & 12 & 2 & Calcareous & friable & rough & sharp & Present $(8 \%)$ \\
\hline
\end{tabular}

The percentage of the presence of subcortex in the samples per each mine population is presented in parenthesis 
Fig. 6 Chert structures of Peschici and Scaglia Fms.
Arciprete

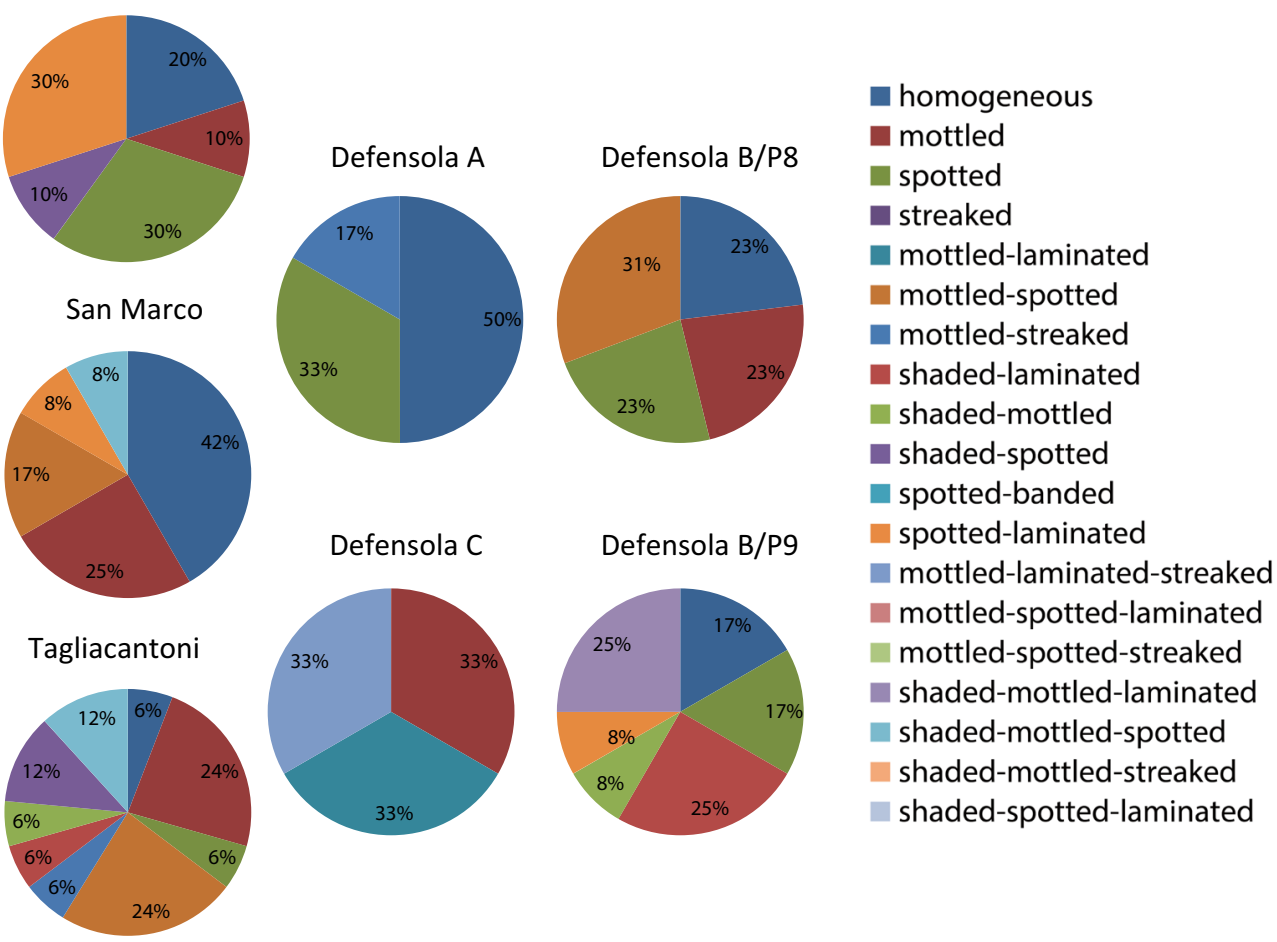

mines of Defensola are characterized by a larger variability. Except for Defensola A, all these mining structures are located in the Peschici Fm., and approximately half of the samples show a subcortex. The cortexes of the samples from Maiolica are essentially devoid of subcortexes. Scaglia Fm. samples have thin cortexes.

\section{Structure}

Observations with the naked eye for each chert sample showed visible structures on the fresh surfaces. The list of visual descriptions is presented in Figs. 6 and 7. Except for Defensola C, all Peschici Fm. mines showed variable numbers
Fig. 7 Chert structures of Maiolica Fm.

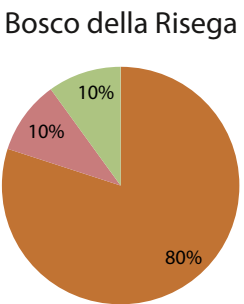

Coppa di Rischio

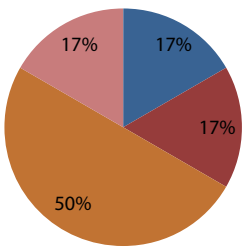

Cruci

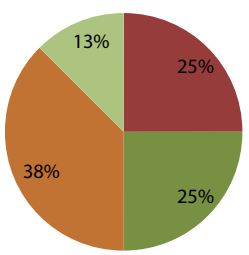

Mastro Tonno

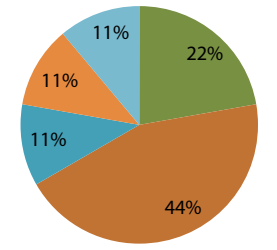

Valle Sbernia/Guariglia

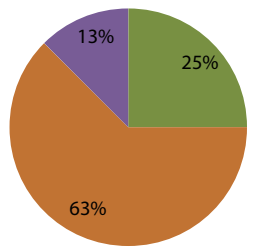

Martinetti

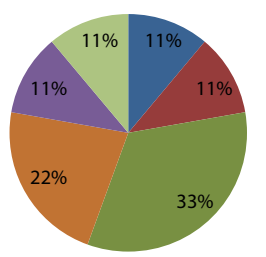

Valle Guariglia I

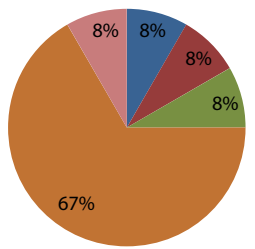

Carmine

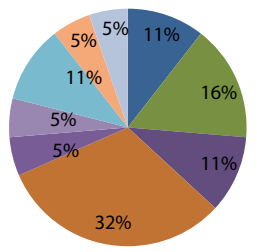

a homogeneous

mottled

spotted

streaked

mottled-laminated

mottled-spotted

mottled-streaked

shaded-laminated

- shaded-mottled

- shaded-spotted

npotted-banded

spotted-laminated

mottled-laminated-streaked

mottled-spotted-laminated

mottled-spotted-streaked

a shaded-mottled-laminated

- shaded-mottled-spotted

shaded-mottled-streaked

shaded-spotted-laminated 
of homogeneous samples. Common structures were the spotted and mottled varieties as well as associations of such features with laminated and shaded ones. The streaked structures were infrequent. The chert of Tagliacantoni (Scaglia Fm.) was characterized by a larger variability of structures, with relatively abundant mottled and mottled-spotted structures. A completely different situation was observed for the Maiolica Fm., where most of the samples appeared mottled-spotted and homogeneous samples were infrequent. In addition, there were frequent varieties of laminated and shaded samples. Occasionally, streaked and banded structures were also observed.

\section{Texture}

Sorting and percentages of inclusions observable under stereomicroscope were recorded for each sample with the goal of obtaining a trend for each mine (Electronic supplementary material (S1)). Table 4 sums the results obtained.

All the mines of Defensola showed common textural features except for Defensola C, which differed for sorting, while San Marco had greater variability for both sorting and percentage of inclusions. The Scaglia Fm. mine of Tagliacantoni had poor sorting and an intermediate number of inclusions. All Maiolica Fm. mines showed poor sorting, while the percentages of allochems generally ranged between 10 and 50. The percentages of inclusions exceeded 50 for Mastro Tonno, Cruci and Valle Guariglia I. Furthermore, all Gargano samples were translucent; thus, translucence was not included in the data processing due to the homogeneity of information.

Table 4 More frequent sorting and fraction of the chert samples per each mine population

\begin{tabular}{lll}
\hline Mine & Sorting & Fraction (\%) \\
\hline Arciprete & Moderate & $10-50$ \\
Defensola A & Moderate & $10-50$ \\
Defensola B/P9 & Moderate & $10-50$ \\
Defensola B/P8 & Moderate & $10-50$ \\
Defensola C & Poor & $10-50$ \\
San Marco & Well & $<10$ \\
Bosco della Risega & Poor & $10-50$ \\
Carmine & Poor & $10-50$ \\
Coppa di Rischio & Poor & $10-50$ \\
Cruci & Poor & $>50$ \\
Martinetti & Poor & $10-50$ \\
Mastro Tonno & Poor & $>50$ \\
Valle Guariglia I & Poor & $>50$ \\
Valle Sbernia/Guariglia & Poor & $10-50$ \\
Tagliacantoni & Poor & $10-50$ \\
\hline
\end{tabular}

\section{Micropaleontology}

Stereomicroscopic observations executed on all the exposed surfaces of the samples allowed evaluation of the main biotypes preserved in the Gargano chert. Although various bioclasts have been recognized in many cases, the database of microscopic observations was filtered by choosing diagnostic criteria for each geological formation or mining structure examined. A detailed micropaleontological analysis on thin sections of the geological samples, which certainly would improve such recognition for an assessment of paleoenvironmental reconstruction, would be beyond the scope of this study. The nondestructive method applied in this study provided identification of the main taxonomic groups occurring in the samples while preventing classification at a lower systematic rank. Within the analysed samples, the microfossil content mainly consisted of radiolarians (mainly Spumellaria), sponge spicules and foraminifers (mainly benthic), as shown in Fig. 12 .

Mean abundances of biotypes of each mine showed an unequal distribution of radiolarians and foraminifers among the samples. Sponge spicules represented the most important component in Peschici Fm. mines, as well as foraminifers, although with variable abundances (Fig. 8), while radiolarians were sporadic or absent, excluding Tagliacantoni. Tagliacantoni seemed to be similar to the Scaglia and Maiolica Fms. in its radiolarian content. This trend changed for Maiolica mines, where radiolarians were a major component, followed by sponge spicules and foraminifers.

\section{Geochemistry}

Chemical analyses carried out on the matrix portion of each sample allowed the measurement of eight elements (Fe, K, Sr, Rb, Ni, Mn, As and Ba). In Fig. 9, chemical composition trends are shown by means of line graphs connecting the medians determined in each mine for the selected elements. Median values were used instead of means since median values are less sensitive to outliers than mean values. The Peschici Fm. mines show quite similar distributions for all elements (Fig. 9a). In the same plot, the Tagliacantoni mine (Scaglia Fm.) diverges because of lower As values. Compared to those of the Peschici Fm., the chemical trends of the Maiolica Fm. mines were more complex. Valle Guariglia I and Valle Sbernia/Guariglia mines showed lower values for $\mathrm{Ni}$, and Mastro Tonno presented lower values for $\mathrm{Mn}$, while As was poorer in the Cruci mine. Usually, high contents of $\mathrm{Ba}$ characterized Maiolica Fm. mines, with the exception of Carmine and Mastro Tonno. 
Fig. 8 a, b Waste sample of Defensola A mine. c, d Waste sample of San Marco mine. Foraminifers and sponge spicules are well visible in both cases. $\mathbf{e}, \mathbf{f}$ Waste sample of Valle Guariglia I mine. Common poor sorting is well visible in subpanel f. Two red radiolarians are evident in microphotographs $\mathbf{g}$ and $\mathbf{h}$
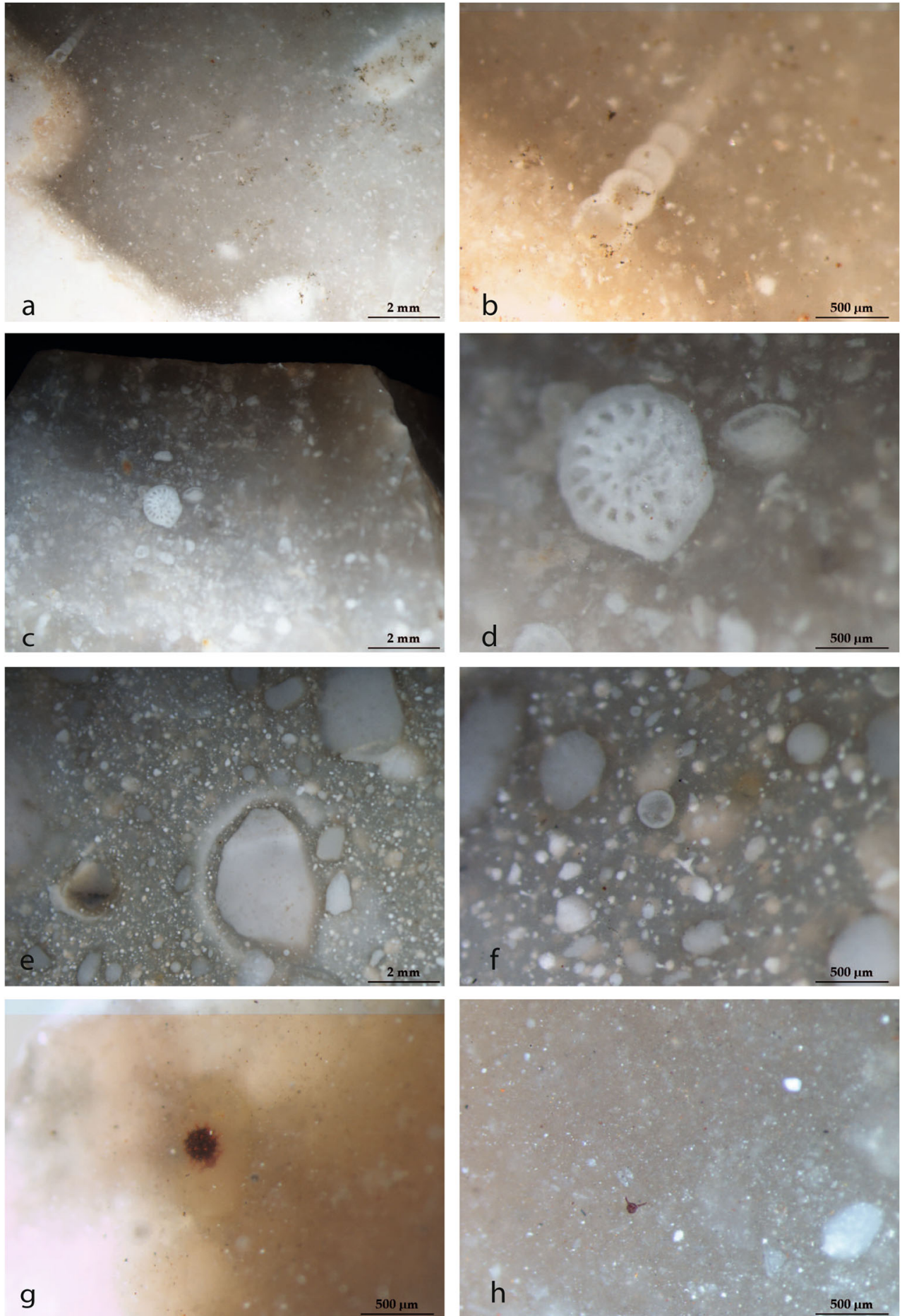

\section{Surface features}

\section{Colour}

To evaluate colour differences among the Gargano mines, the means of the three colorimetric coordinates for each mine were plotted as shown in Fig. 10.
In general, noticeable grouped samples in the colour space were characterized by low positive values of $a^{*}$ and $b^{*}$ and lightness values $\left(L^{*}\right)$ lower than 50 .

Commonly, the colour trend of Gargano chert was mainly grayish to light yellow, and samples from the Maiolica Fm. appeared less variable. Numerous chert samples from the Peschici Fm. diverged from the 
a

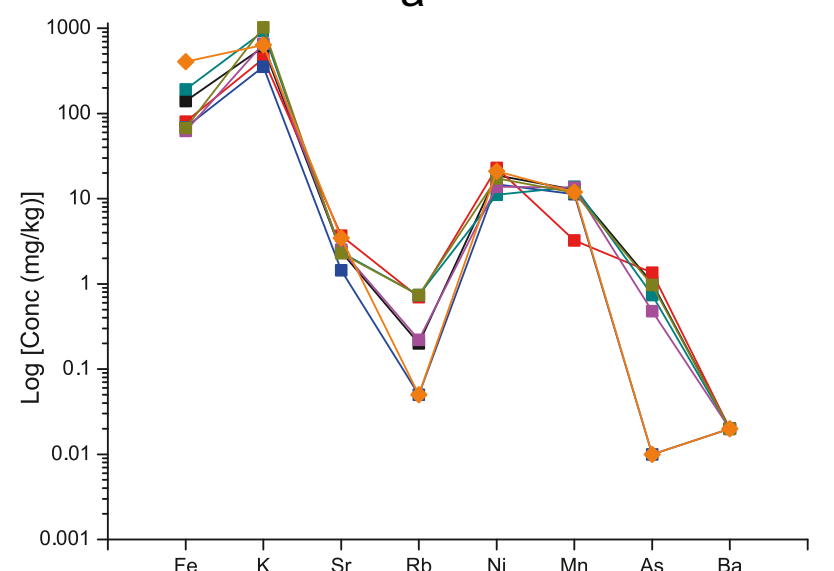

Peschici Fm:

$\longrightarrow$ Arciprete

$\rightarrow$ Defensola A

Scaglia Fm:

$\longrightarrow$ Tagliacantoni

b

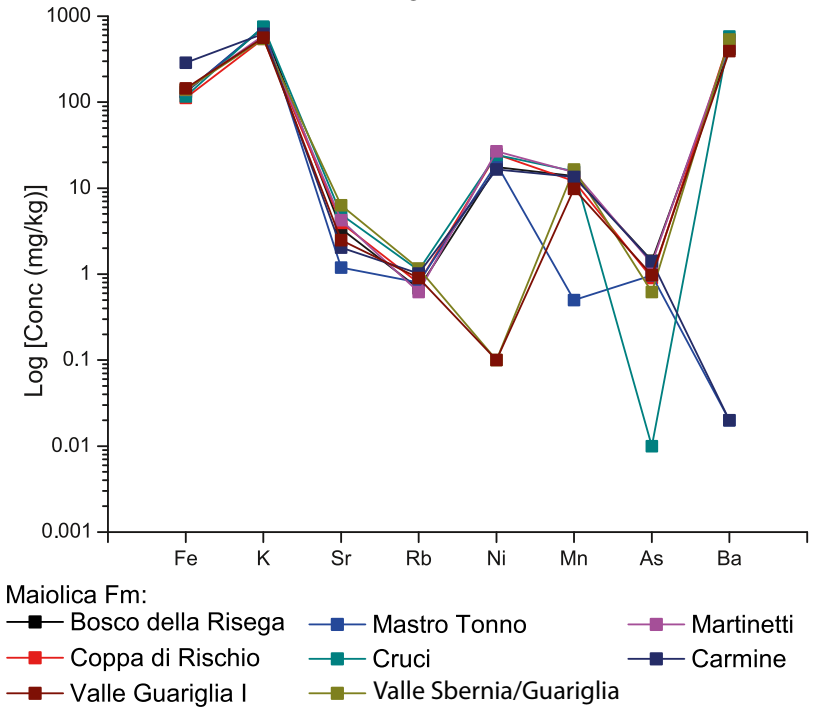

Fig. 9 Median values and geochemical patterns of Peschici Fm. and Scaglia Fm. mines (a) and Maiolica Fm. mines (b)

aforementioned trend, having greater values of $a^{*}$ and $b^{*}$ (orange hues). The Peschici Fm. mines had $L^{*}$ mean values greater than 40 , and only the two mines of Defensola A and C had darker samples. Darker colours were associated with Maiolica Fm. mines, except for Mastro Tonno samples. Red-orange colour distinguished the Tagliacantoni mines, which are located at the stratigraphic limit between the Scaglia and Peschici Fms. Two mines in the Maiolica Fm. (i.e. Carmine and Martinetti) exhibited reddish chert. The chert from Defensola B/P9 and Defensola $\mathrm{C}$ mines presented pale grayish colours. Three mines showed a greater range of colours: Arciprete, Defensola B/P8 and San Marco. The mines in the Maiolica Fm. generally presented less variable colours, except for Carmine, Martinetti and Valle Guariglia I.

\section{Fracture}

Gargano mines generally showed samples with a conchoidal fracture, which made this chert a raw material of high quality for knapping. The cherts from the Cruci and Martinetti mines presented higher incidences of uneven fractures, probably as a consequence of tectonic deformations. These two mines included only a few samples with mottled-spotted-streaked structures. Arciprete and Defensola B/P9 included several samples with subconchoidal fractures. Arciprete and Defensola B/P9 showed more samples with laminated and shaded structures.

\section{Discussion and conclusions}

The mixed data matrix obtained by NM-PCI consists of 33 variables. Sixteen of them are binary, six ordinal and 11 continuous, for a total of 155 cases. A rearrangement of the data and a transformation were performed before proceeding with the multivariate statistics. Based on the dissimilarity matrix, the most similar pair of data are those of the Cruci mine, while the most dissimilar pair are two samples from the Tagliacantoni and San Marco mines. Running the cluster analysis procedure with PAM, the complex of data should be split into 15 (i.e. $k$ ) clusters and relative medoids, which correspond to the number of mines investigated. However, this choice does not mean that 15 is the best number of clusters for the data set. For this reason, the silhouette width was used as an internal validation tool to choose the most appropriate number of clusters.

The silhouette plot (Fig. 11) suggested 12 or 13 groups (given the close high values of the silhouette width). For a more detailed discussion, the PAM algorithm was run two times with both 13 and 15 as $k$ values, and the respective silhouette plots were compared. The Dunn and Rand indices were also calculated.

The Dunn index was unvaried for both solutions, while the silhouette index and Rand index increased when 13 clusters were chosen, and the number of misclassified cases decreased to two. Therefore, the clustering structure that best represented the mines data set was the one with $k=13$. Each mine sample was allocated to the most similar medoid, and the final output showed 13 clouds of points or clusters. Figure 12 shows the output of the routine; the ellipses of concentration include 95\% of samples for each identified cluster; each mine is indicated with a different marker.

The crosschecking of the outcome highlights that nine mines were well identified; the mining complex of Defensola, Arciprete and San Marco is compact. Defensola 

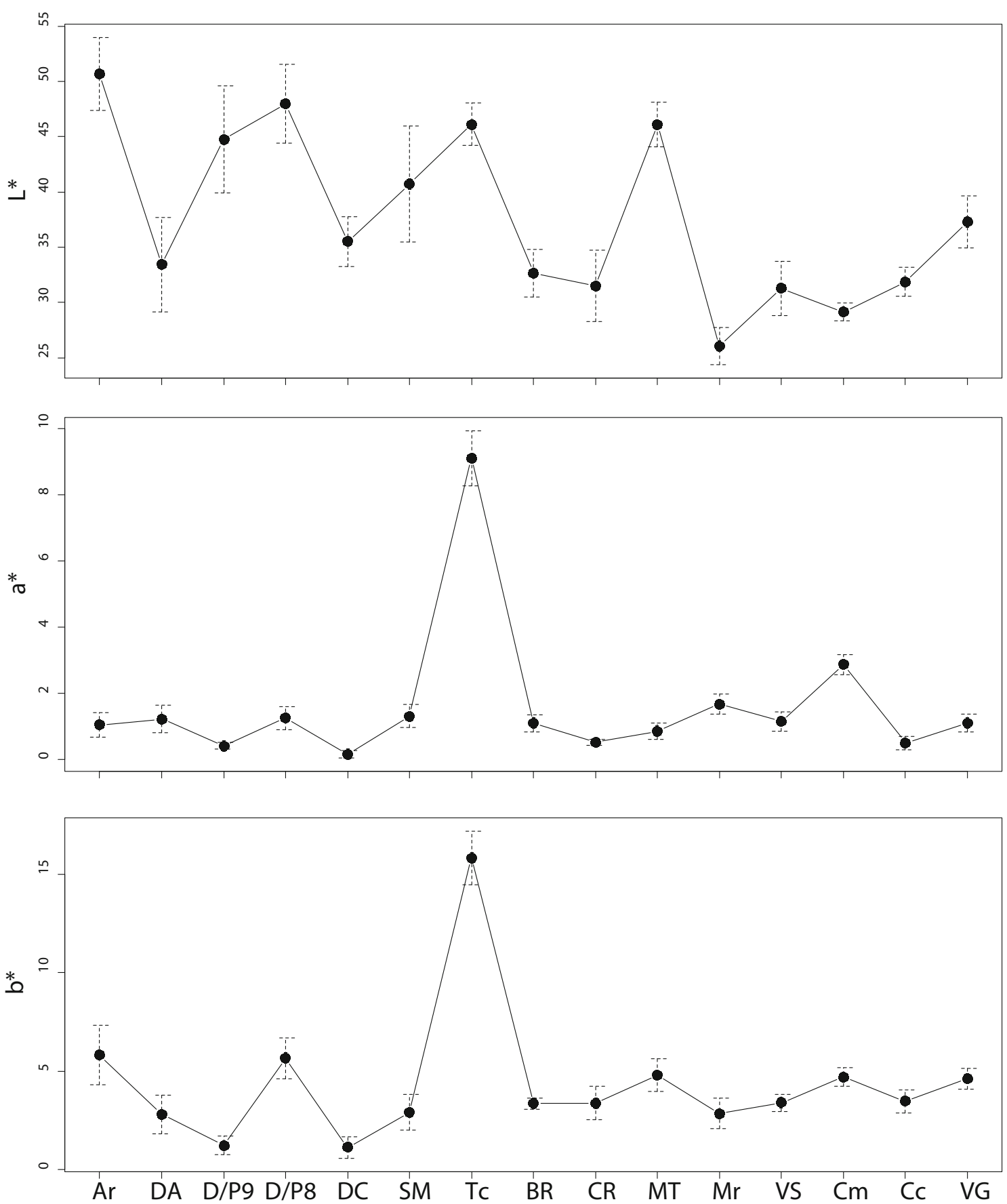

Fig. 10 The mean and the standard deviation of each colour coordinate $\left(L^{*}, a^{*}, b^{*}\right)$ of the chert from 15 mines examined. (Ar, Arciprete; DA, Defensola A; D/P9, Defensola B/P9; D/P8, Defensola B/P8; DC,

Defensola C; SM, San Marco; Tc, Tagliacantoni; BR, Bosco della Risega; CR, Coppa di Rischio; MT, Mastro Tonno; Mr, Martinetti; VS, Valle Sbernia/Guariglia; Cm, Carmine; Cc, Cruci; VG, Valle Guariglia I)

$\mathrm{C}$ is isolated from other Vieste district mines belonging to the Peschici Fm. Near the cluster of Defensola C, the samples from Tagliacantoni and Carmine are arranged in two clusters. To the left of these two clusters, the cluster of Mastro Tonno is well centered, while the clusters of Cruci and Valle Guariglia I overlap. The four Maiolica Fm. mines overlap (i.e. Bosco della Risega, Coppa di Rischio, Martinetti and part of the
Valle Sbernia/Guariglia samples), and the medoid in this cluster is a sample from Bosco della Risega.

Two blue ellipses (Fig. 12, clusters 11 and 3) contain a few scattered samples; in one case, these samples belong to Defensola B/P9, and in the other case, the cluster includes a few samples from Cruci, Martinetti, Valle Sbernia/Guariglia and only one from Valle Guariglia I (the medoid is a sample 
Fig. 11 Silhouette widths of the outcome of PAM. It is observed that the higher values of silhouette width correspond to 12 and 13 clusters

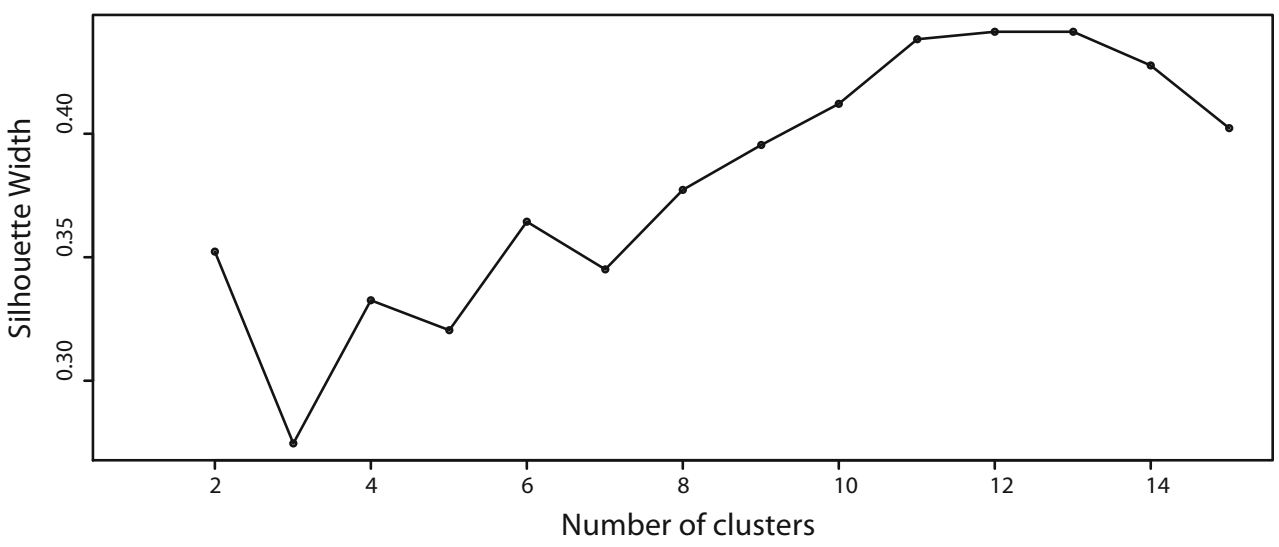

from Valle Sbernia/Guariglia). The samples misclassified in the silhouette width are one from Tagliacantoni, attributed to Carmine (yellow square closer to Carmine ellipse), and one from Cruci, which falls within the blue ellipse. The mines that show greater variability are those spread in two clusters: Defensola B/P9, Martinetti and Valle Sbernia/Guariglia. As a whole, the geological formations are well distinguished. The samples from the Peschici Fm. are located on the right side of the graph, while Maiolica samples are grouped on the left side. The Peschici Fm. is characterized by greater variability of chert features, making it possible to discriminate individual mines, as in the case of the Defensola complex. On the other hand, cherts from the Maiolica mines seem to be very similar, especially in the cases of Bosco della Risega and Coppa di Rischio. The strength of the classification output is to define with a higher resolution the inner variability of the formations. Two cases from Valle Guariglia I and Cruci, isolated from other Maiolica mines, can be explained by their position close to the heteropic transition between the Maiolica and Marne a Fucoidi Fms. As evidenced by the output, different features characterized this geological area.

Similarly, the Tagliacantoni mine (attributed to the Peschici Fm. from bibliographic sources) is well isolated from other Peschici mines, since it falls in the borderline area between the Peschici and Scaglia Fms. (see Figs. 1 and 2). Very different chert features lead to attributing the Tagliacantoni mine to the Scaglia rather than to the Peschici Fm. The Carmine mine, attributed to the Maiolica Fm., is geographically and compositionally well isolated from other Maiolica mines investigated here (located in the higher part of the same formation). This mine is the only one identified in the lower part of the Maiolica Fm. in the southern part of the Gargano Promontory. Finally, variations in the sedimentary environmental conditions may explain the isolated cases of Mastro Tonno and Defensola C. After the results were

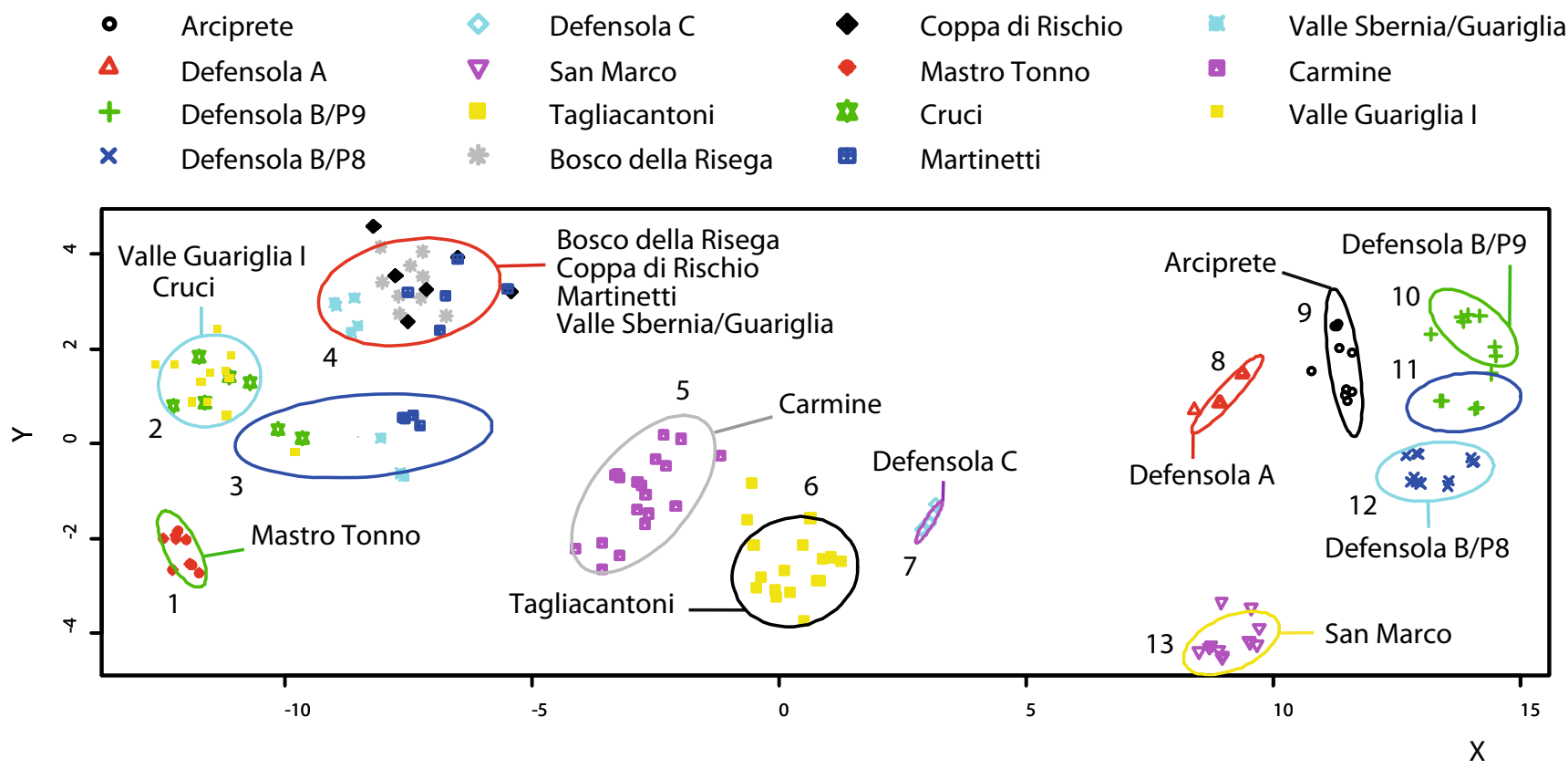

Fig. 12 Output of PAM classification. The investigated mines are distributed in 13 clusters as indicated by concentration ellipses (95\% of samples) and relative number (see text). Each marker indicate a specific mine 
obtained from the NM-PCI on the cherts, the original geological attributions of the Valle Guariglia I, Cruci and Tagliacantoni mines were reconsidered, and inter- and intraformational differences were highlighted with greater resolution.

Generally, in each geological formation, specific structural and textural features were observed. Homogeneous, spotted and mottled features were found within the Peschici Fm., mottled and mottled-spotted within the Scaglia Fm. and mottled-spotted into the Maiolica Fm. Moderate sorting characterized the Peschici Fm., and poor sorting characterized the Maiolica Fm.

Defensola C (poor) and San Marco (well) diverged from these sorting trends. The percentages of inclusions generally fell in the range between 10 and 50. Lower amounts were observed for San Marco and higher for Cruci, Valle Guariglia I and Mastro Tonno.

The colour of Gargano chert investigated here is quite homogeneous, dominated by grayish tones. Peschici Fm. chert appears to be lighter than Maiolica Fm. chert. Color variables make it possible to distinguish the reddish-orange chert of the Tagliacantoni mine and Carmine from those of other mining facilities. The geochemical trends of Gargano mines are partially different; Ba values separate the Peschici Fm. context from that of the Maiolica Fm., but the Carmine and Mastro Tonno mines are exceptions. The higher values of $\mathrm{Fe}$ observed for Tagliacantoni and Carmine explain the reddish colour of the samples. Barium concentrations are comparable with the results obtained by Volterra et al. (2002) for chert samples from seven Gargano mines, while some variations in the potassium contents are found for Arciprete and Defensola A, which show higher median values.

Abundances of radiolarians and foraminifers vary significantly between the Peschici and Maiolica Fms. Sponge spicules are very plentiful for all Peschici Fm. mines (minor amounts at Defensola B/P9), while the Maiolica Fm. shows greater variability of spicules (major amounts for Mastro Tonno and Carmine, minor amounts for Cruci and Valle Sbernia/ Guariglia and traces for Bosco della Risega, Coppa di Rischio and Martinetti). Radiolarians and sponges are abundant in Tagliacantoni (Scaglia Fm.), with minor amounts of foraminifers.

Structural features, micropaleontological contents and geochemical data individually allow the separation of two main groups: the mines of the Peschici Fm. from those of the Maiolica Fm. Each group shows a strong overlap of mines, and only a few mines are characterized by specific traits: Defensola $\mathrm{C}$ for structure, Tagliacantoni and Martinetti for structure and colour and Carmine for structure, colour and micropaleontological contents. The use of PAM provides insight into the mixed data structure at the level of each mine's inner variability. Concentration ellipses allow the separation of nine mines out of the 15 examined, whereas strong similarities caused the overlapping of some Maiolica Fm. mines.
Structure, texture, geochemistry and micropaleontology have strong weights on the separation between the Peschici and Maiolica Fms. The Scaglia Fm. appears to have intermediate features between those of the Maiolica and Peschici Fms. Colour coordinates isolate Tagliacantoni and Carmine from all other mines. The Peschici Fm. shows greater inner variability than the Maiolica Fm., where four mines are grouped in the same cluster. Structure, sorting and percentages of inclusions separate Defensola C and San Marco from other Vieste district mines and Valle Guariglia I, Cruci and Mastro Tonno from other Peschici district mines.

Compared to the cluster analysis results of Volterra et al. (2002) obtained from seven Gargano mines-Coppa di Rischio, Valleguariglia (namely, Valle Sbernia/Guariglia), Martinetti, Defensola A, Defensola B, Arciprete and Tagliacantoni- using geochemical data exclusively ( $\mathrm{Al}, \mathrm{Ba}, \mathrm{K}$, $\mathrm{Mg}, \mathrm{Na}, \mathrm{P}$ and Ti), our NM-PCI method separated with greater resolution specific deposits such as Tagliacantoni. The application of the NM-PCI method to Gargano cherts shed light on the distinctive features of the chert mines and helped solve problems about the stratigraphic positions of some mines.

The modifications made to the multiparametric protocol introduced by Tarantini et al. (2016) implemented new features (i.e. ED-pXRF, texture and micropaleontological data) and a multivariate statistical methodology to process mixed data (PAM algorithm). The results obtained in this case study allowed a first evaluation of the NM-PCI protocol.

The NM-PCI protocol aims at improving the traditional methodologies used in the archaeological field (e.g. naked eyes and microscopic descriptions), generally coupled with geochemical characterisation of chert samples. Scientific efforts were focused on the standardisation of analytical procedures and the improvement of description strategies for visible appearance and petrographical features, based on portable nondestructive devices measuring colour and geochemical composition. Moreover, specific statistical strategies allowed merging the information gathered from different categories of data for a more effective representation of the data structure and classification.

The strength of the approach proposed here lies in the nondestructivity of the procedure employed, the rapidity of data acquisition and the low cost of analyses, in line with archaeological requirements. The analytical protocol can be applied in situ, with portable laboratory equipment such as digital microscopy, stereomicroscopy, spectrophotometry and pXRF instrumentation, providing rapid data acquisition in the field or in the laboratory. The appreciable results obtained for the Gargano mining context motivate the implementation of NM-PCI as a promising tool for provenance studies of archaeological cherts. Additional multivariate statistical techniques could be used to compare the results of investigations. Over $60 \%$ of the examined mines were distinguishable from one other, with the remainder overlapping in two clusters. A more in-depth observation of microfossils (e.g. 
distinction between planktonic and benthic foraminifera) could improve the separation of mines, and an estimation of additional trace element concentrations with other chemical methods (e.g. LA-ICP-MS or LIBS) could be useful to improve the resolving power of cluster analysis.

Acknowledgments This paper is part of the $\mathrm{PhD}$ thesis of one of the authors (ED). Attilio Galiberti is kindly acknowledged for providing some of the sample analysed here. We also thank Jehanne Affolter for her help in the micropaleontological investigations. The ED-pXRF analyses were carried out at the 'Micro X-ray Lab' of the University of Bari Aldo Moro, supported by Regione Puglia (Programma Operativo Regione Puglia-FERS 2000-2006-Risorse Liberate-Obiettivo Convergenza). The authors gratefully acknowledge the Italian Ministry of Education, University and Research (MIUR Funds-PhD in Geosciences, Bari University).

\section{References}

Affolter J (1994) Les microfossiles des silex. Rapport de stage FNRS, Bruxelles

Affolter J (2002) Provenance des silex préhistoriques du Jura et des régions limitrophes. Service et Musée cantonal d'archéologie, Lousanne

Affolter J, Bintz P, Bressy C (1999) Analyse et circulation des matières premières siliceuses au Mésolithique et au Néolithique ancien dans les Alpes du Nord. In: Beeching A (ed) Circulations et identités culturelles alpines à la fin de la préhistoire - Matériaux pour une étude, Programme CIRCALP 1997-1998, Travaux du Centre d'Archéologie Préhistorique de Valence, $n^{\circ}$ 2, Valence, pp 175-181

Allard P, Bostyn F, Giligny F, Lech J (2008) Flint mining in prehistoric Europe: interpreting the archaeological record, BAR International Series. Archaeopress, Oxford, p 1891

Ahler SA (1983) Heat treatment of Knife River flint. Lithic Technol 12:1-8 Ambrose SH (2001) Paleolithic technology and human evolution. Science 291(5509): 1748-1753

Aspinall A, Feather SW (1972) Neutron activation analysis of prehistoric flint mine products. Archaeometry 14(1):41-53

Basili R, Di Lernia S, Fiorentino G, Galiberti A (1995) Review of prehistoric chert mines in the "Gargano" promontory (Apulia, Southern Italy). Archeologia Polona 33:414-436

Baxter MJ (1994) Exploratory multivariate analysis in archaeology. Edinburgh University Press, Edinburgh

Berger-Schunn A (1994) Practical color measurement. Wiley, New York

Bernoulli D (1972) North Atlantic and Mediterranean Mesozoic facies: a comparison. Initial Rep Deep Sea Drill Proj 11:801-871

Bertola S (2011) The flints of Southern Alps (Non Valley, Italy) provenance found in the Mesolithic site of Ullafelsen (Sellrain, Tyrol). In Schäfer D (ed) Das Mesolithikum-Projekt Ullafelsen (Teil 1). Mensch und Umwelt im Holozän Tirols, 1. Philipp von Zabern, Innsbruck, pp 463-505

Bertola S (2012) Approccio micropaleontologico discriminante per riconoscere la provenienza alpina o appenninica delle selci della scaglia rossa (Italia centro-settentrionale). Bulletin du Musée d'Anthropologie préhistorique de Monaco 52:17-27

Bhat A (2014) K-medoids clustering using partitioning around medoids for performing face recognition. International Journal of Soft Computing, Mathematics and Control 3(3):1-12
Binder D, Perlès C, Inizan ML, Lechevallier M (1990) Stratégies de gestion des outillages lithiques au Néolithique. Paléo 2:257-283

Boggs Jr S (2009) Petrology of Sedimentary Rocks. Cambridge University Press

Borcard D, Gillet F, Legendre P (2011) Spatial analysis of ecological data. In: Numerical ecology with R. Springer, New York, pp 227-292

Borgomano JRF (2000) The Upper Cretaceous carbonates of the Gargano-Murge region, southern Italy: a model of platform-tobasin transition. AAPG Bull 84(10):1561-1588

Bosellini A, Morsilli M (1997) A Lower Cretaceous drowning unconformity on the eastern flank of the Apulia Platform (Gargano Promontory, southern Italy). Cretac Res 18(1):51-61

Bosellini A, Morsilli M, Neri C (1999) Longterm event stratigraphy of the Apulia Platform margin (Upper Jurassic to Eocene, Gargano, southern Italy). J Sediment Res 69(6):1241-1252. https://doi.org/10. 2110/jsr.69.1241

Brajnikov B (1937) Recherches sur la formation appelée "Argiles à silex" dans le bassin de Paris. Revue de Géographie Physique et Géologie Dynamique 10(1-2):7-130

Bressy C (2002) Caractérisation et gestion du silex des sites mésolithiques et néolithiques du nord-ouest de l'arc alpin. Une approche pétrographique et géochimique Dissertation, Université de Provence-Aix-Marseille I

Bressy C (2003) Caractérisation et gestion du silex des sites mésolithiques et néolithiques du Nord-Ouest de l'arc alpin. Un approche pétrographique et géochimique. BAR International Series 1114, Archaeopress, Oxford

Cailleux A, Taylor G (1963) Code Expolaire. Edit. Boubée et Cie, Paris

Caldara M, Caroli I, Simone O (2008) Holocene evolution and sea-level changes in the Battaglia basin area (eastern Gargano coast, Apulia, Italy). Quat Int 183(1):102-114

Caroli I, Caldara M (2007) Vegetation history of Lago Battaglia (eastern Gargano coast, Apulia, Italy) during the middle-late Holocene. Veg Hist Archaeobotany 16(4):317-327

Chasteignier A de (1868) L'Age de pierre dans les landes de le Gironde, découverte des ateliers de fabrication des pointes de flèches et de l'origine du silex employé. Revue Archéologique 18:95-99

Craig N, Speakman RJ, Popelka-Filcoff RS, Glascock MD, Robertson JD, Shackley MS, Aldenderfer MS (2007) Comparison of XRF and PXRF for analysis of archaeological obsidian from southern Peru. J Archaeol Sci 34:2012-2024

Cobianchi M, Luciani V, Bosellini A (1997) Early Cretaceous nannofossils and planktonic foraminifera from northern Gargano (Apulia, southern Italy). Cretac Res 18(2):249-293

Consuegra S, Díaz-del-Río P (2018) Early prehistoric flint mining in Europe: a critical review of the radiocarbon evidence. In: Werra DH, Woźny M (eds) Between history and archaeology. Papers in honour of Jacek Lech. Archeaopress, London, pp 1-8

Damour A (1865) Sur la composition des Haches en pierre trouvées dans les monuments celtiques et chez les sauvages. C R Acad Sci LXI:1-13

Deflandre G (1935) Technique micropaléontologique appliquée à l'étude des silex. Bull Soc fr Microsc, Paris 5(2):76-79

D'Ottavio F (2001) La caratterizzazione chimica della selce delle miniere preistoriche del Gargano. Proposta di un metodo archeometrico basato sulle analisi chimiche eseguite con la tecnica strumentale ICP-AES. Origini 23:111-144

D'Ottavio F, Palmieri A (2005) La circolazione della selce garganica. Caratterizzazione della selce e prospettive di ricerca. In: Galiberti A (ed) Defensola. Una miniera di 7000 anni fa. Protagon, Siena, pp 189-196

D’Ottavio F, Palmieri AM, Volterra E (2000) Provenance studies of prehistoric chert from the Gargano mines and from prehistoric sites of the Tavoliere (Puglia, Italy): sources characterisation and possible attribution. In: Proceedings of the $2^{\text {nd }}$ International Congress "Science and Technology for the Safeguard of Cultural Heritage in the Mediterranean Basin" (Paris 1999), vol. I, Paris, pp 263-266 
De Francesco AM, Crisci GM, Bocci M (2008) Non-destructive analytic method using XRF for determination of provenance of archaeological obsidians from the mediterranean area: a comparison with traditional XRF methods. Archaeometry 50:337-350

Di Lernia S, Fiorentino G, Galiberti A (1990) «Gargano prehistoric flint mines project»: the state of research in the Neolithic Mine of Defensola-Vieste (Italy). Origini 15:175-199

Di Lernia S, Fiorentino G, Galiberti A, Basili R (1997) Topography of Gargano mining sites between geological context and quarrying techniques: a preliminary investigation. In: Ramos-Millán A, Bustillo MA (eds) Siliceous rocks and culture. Universidad de Granada, Granada, pp 195-209

Dunham RJ (1962) Classification of carbonate rocks according to depositional texture. In: Ham WE (ed) Classification of carbonate rocks, American Association of Petroleum Geologists Memoir 1, pp 108-121

Dunn JC (1974) Well-separated clusters and optimal fuzzy partitions. J Cybernetics 4(1):95-104

Eberli GP, Bernoulli D, Sanders D, Vecsei A (1993) From aggradation to progradation: the Maiella Platform, Abruzzi, Italy. In: Simo T, Scott RW, Masse JP (eds) Cretaceous carbonate platforms. American Association of Petroleum Geologists Memoir 56, pp 213-232

Everitt BS, Landau S, Leese M, Stahl D (2011) Cluster analysis. In: Wiley series in probability and statistics, 5th edn. Wiley and Sons, New York

Fernandes P (2012) Itinéraires et transformations du silex: une pétroarchéologie refondée, application au Paléolithique moyen. Dissertation, Université de Bordeaux 1

Fernandes P, Le Bourdonnec FX, Raynal JP, Poupeau G, Piboule M, Moncel MH (2007) Origins of prehistoric flints: the neocortex memory revealed by scanning electron microscopy. Comptes Rendus Palevol 6(8):557-568

Fernandes P, Raynal JP (2006) Pétroarchéologie du silex: un retour aux sources. Comptes Rendus Palevol 5(6):829-837

Fernandes P, Raynal JP (2010) Silex: une pétroarchéologie refondée. Les cahiers de Géopré - Silex et territoires préhistoriques. Avancées des recherches dans le Midi de la France 1:68-81

Folk RL, Weaver CE (1952) A study of the texture and composition of chert. Am J Sci 250(7):498-510

Forster N, Grave P (2012) Non-destructive PXRF analysis of museum curated obsidian from the Near East. J Archaeol Sci 39:728-736

Galiberti A (2005) Defensola. Una miniera di selce di 7000 anni fa. Protagon, Siena

Gauthier G, Burke AL, Leclerc M (2012) Assessing XRF for the geochemical characterization of radiolarian chert artifacts from northeastern North America. JArchaeol Sci 39(7):2436-2451

Gelato G (1994) Petrografia, mineralogia e geochimica delle selci del Gargano nord-orientale. Dissertation, Università degli Studi di Bari

Glascock MD (2012) Comparison and contrast between XRF and NAA: used for characterization of obsidian sources in Central Mexico. In: Shackley MS (ed) X-ray fluorescence spectrometry (XRF) in geoarchaeology. Springer, New York, pp 161-192

Gower JC (1971) A general coefficient of similarity and some of its properties. Biometrics 27:857-871

Greensmith J (2012) Petrology of the Sedimentary Rocks. Springer Science \& Business Media

Hennig C (2014) Package Fpc: flexible procedures for clustering R. version 2.2-1. https://cran.r-project.org/web/packages/fpc/fpc.pdf

Hess SC (1996) Chert provenance analysis at the Mack Canyon site, Sherman County, Oregon: an evaluative study. Geoarchaeology 11(1):51-81

Hughes RE (1983) Exploring diachronic variability in obsidian procurement patterns in Northeast California and South Central Oregon: geochemical characterization of obsidian sources and projectile points by energy dispersive X-ray fluorescence anthropology. Dissertation, University of California, Davis
Hughes RE (1988) The Coso Volcanic Field reexamined: implications for obsidian sourcing and hydration dating research. Geoarchaeology 3 : 253-265

Izenman AJ (2008) Modern multivariate statistical techniques. Regression, classification and manifold learning. Springer, New York

James MA, Bailey J, D'Auria JM (1996) A volcanic glass library for the pacific northwest: problems and prospects. Can J Archaeol 20:93-122

James G, Witten D, Hastie T, Tibshirani R (2013) An introduction to statistical learning, vol 112. Springer, New York

Journal of Lithic Studies. (2014) Volume 1, Number 1. https://doi.org/10. 2218/jls.v1i1

Journal of Lithic Studies. (2016) Volume 3, Number 2. https://doi.org/10. 2218/jls.v3i2

Kaufman L, Rousseeuw PJ (1990) Partitioning around medoids (program PAM). In: Kaufman L, Rousseeuw PJ (eds) Finding groups in data: an introduction to cluster analysis. John Wiley \& Sons, Hoboken, pp $68-125$

Kassambara A (2017) Practical guide to cluster analysis. In: R: unsupervised machine learning, vol 1 STHDA

Klein C, Hurlbut CS, Dana JD (1993) Manual of mineralogy. John Wiley $\&$ Sons, New York

Korlin G, Weisgerber G (2006) Stone Age, Mining Age. Der Anschnitt 19. Deutsches Bergbau Museum, Bochum

Krijthe JH (2015) Rtsne: T-distributed stochastic neighbor embedding using a Barnes-hut implementation. https://github.com/jkrijthe/ Rtsne

Krukowski S (1939) Paleolit Polski. Drukarnia Uniwersytetu Jagiellońskiego, Cracovie

Léa V (2004) Les industries lithiques du Chasséen en Languedoc oriental. Caractérisation par l'analyse technologique. BAR International Series 1232, Archaeopress, Oxford

Luedtke BE (1978) Chert sources and trace element analysis. Am Antiq 43:413-423

Luedtke BE (1979) The identification of sources of chert artifacts. Am Antiq 44:744-757

Luedtke BE (1992) An archaeologist's guide to chert and flint. Cotsen Institute of Archaeology Press, Los Angeles

Maaten LVD, Hinton G (2008) Visualizing data using t-SNE. J Mach Learn Res 9:2579-2605

Maechler M, Rousseeuw P, Struyf A, Hubert M, Hornik K (2018) Cluster: cluster analysis basics and extensions. $\mathrm{R}$ package version 2.0.7-1. https://cran.r-project.org/web/packages/cluster/cluster.pdf

Malyk-Selivanova N, Ashley GM, Gal R, Glascock MD, Neff H (1998) Geological-geochemical approach to "sourcing" of prehistoric chert artifacts, northwestern Alaska. Geoarchaeology 13(7):673-708

Masson A (1981) Pétroarchéologie des roches siliceuses. Intérêt en Préhistoire, Thèse de troisième cycle, Université Lyon I

Matthew AJ, Woods AJ, Oliver C (1991) Spots before the eyes: new comparison charts for visual percentage estimation in archaeological material. Recent developments in ceramic petrology 81:211-263

McLaren K (1976) XIII - the development of the CIE 1976 (L* a* b*) uniform colour space and colour difference formula. J Soc Dye Colour 92(9):338-341

MacQueen J (1967) Some methods for classification and analysis of multivariate observations. In: Proceedings of the fifth Berkeley symposium on mathematical statistics and probability (Vol. 1, No. 14), pp 281-297

Morsilli M (2011) Introduzione alla Geologia del Gargano. In: Tarantini M, Galiberti A (eds) Le miniere di selce del Gargano VI-III millennio a.C. Alle origini della storia mineraria europea.. All'Insegna del Giglio, pp 17-27

Morsilli M (2016) Sintesi delle conoscenze geologiche e stratigrafiche del Promontorio del Gargano. Geologi e territorio 13(2):15-30

Morsilli M, Bosellini A (1997) Carbonate facies zonation of the upper Jurassic-lower Cretaceous Apulia platform margin (Gargano Promontory, southern Italy). Rivista Italiana di Paleontologia e 
Stratigrafia (Research In Paleontology and Stratigraphy) 103(2): 193-205

Morsilli M, Hairabian A, Borgomano J, Nardon S, Adams E, Gartner GB (2017) The Apulia carbonate platform - Gargano Promontory, Italy (Upper Jurassic-Eocene). AAPG Bull 101(4):523-531

Morsilli M, Rusciadelli G, Bosellini A (2004) The Apulia carbonate platform-margin and slope, Late Jurassic to Eocene of the Maiella Mt. and Gargano Promontory: physical stratigraphy and architecture. In: Field trip guide book-P18, 32nd International Geological Congress, Florence, A.P.A.T, Roma

Munsell AH (1915) Atlas of the Munsell color system. Howland \& Co., Wadsworth

Muntoni IM (2012) Circulation of raw materials, final products or ideas in the Neolithic communities of southern Italy: the contribution of archaeometric analyses to the study of pottery., flint and obsidian. Rubricatum 5:403-411

Nazaroff AJ, Prufer KM, Drake BL (2010) Assessing the applicability of portable X-ray fluorescence spectrometry for obsidian provenance research in the Maya lowlands. J Archaeol Sci 37:885-895

Pawlikowski M (1989) On the necessity of standarization of petrological investigations in archaeology. Prace Archeol 43:7-15

Pelegrin J, Roche H (2017) L'humanisation au prisme des pierres taillées. Comptes Rendus Palevol 16(2):175-181

Perlès C (2004) Les industries lithiques taillées de Franchthi (Argolide, Grèce). Tome III. Du Néolithique ancien au Néolithique final. Excavations at Franchthi Cave. Indiana University Press, Greece, p 13

R Core Team (2018) R: a language and environment for statistical computing. R Foundation for Statistical Computing, Vienna, Austria. http://www.R-project.org/

Rand WM (1971) Objective criteria for the evaluation of clustering methods. J Am Stat Assoc 66(336):846-850

Rellini U, Battaglia R, Baumgaertel E (1930-1931) Rapporto preliminare sulle ricerche paleoetnologiche condotte sul Promontorio del Gargano. Bullettino di Paletnologia Italiana L-LI:43-77

Reynolds AP, Richards G, de la Iglesia B, Rayward-Smith VJ (2006) Clustering rules: a comparison of partitioning and hierarchical clustering algorithms. Journal of Mathematical Modelling and Algorithms 5(4):475-504

Rousseeuw PJ (1987) Silhouettes: a graphical aid to the interpretation and validation of cluster analysis. J Comput Appl Math 20:53-65

de Saint-Venant J. (1911) Tailleries de silex du sud de la France, inventaire des produits exportés aux temps préhistoriques, et carte de leur aire de diffusion. Le Mans, Imprimerie Monnoyer

Sánchez de la Torre M, Le Bourdonnec FX, Dubernet S, Gratuze B, Mangado X, Fullola JM (2017) The geochemical characterization of two long distance chert tracers by ED-XRF and LA-ICP-MS. Implications for Magdalenian human mobility in the Pyrenees (SW Europe). STAR: Science \& Technology of Archaeological Research 3(2):15-27

Sarabia Rogina PM (1990) Approche de l'étude de la distribution stratigraphique du silex de la province de Cantabria (Espagne du Nord). In: Seronie-Vivien MR, Lenoir M. (eds), Le silex de sa genèse à l'outil. Cahiers du Quaternaire 17:141-148

Selivanova N (1984) The methods of source determination of flint for archaeological sites. In: Kunchev KS, Nachev IK (eds) Reports, III seminar in petro-archaeology. Bulgarian Academy of Science, Plovdiv, pp 93-102

Selivanova N (1986) Source location of Neolithic flint materials in the Marveevo-Kurgan District. In: Biro Katalin T. (ed), Papers for the 1st International Conference of Prehistoric Flint Mining and Lithic Raw Material Identification in the Carpathian Basin. Magyar Nomzety Muzeum, Budapest-Sumeg, pp 233-235

Séronie-Vivien M, Seronie-Vivien MR (1987) Les Silex du Mésozoïque nord-aquitain. Approche géologique de l'étude des silex pour servir à la recherche préhistorique, Supplément au tome du Bull. Soc. Linn. Bordeaux XV (suppl.)
Shackley MS (1988) Sources of archaeological obsidian in the southwest: an archaeological, petrological, and geochemical study. Am Antiq 53:752-772

Shackley MS (1995) Sources of archaeological obsidian in the greater American Southwest: an update and quantitative analysis. Am Antiq 60:531-551

Shackley MS (2008) Archaeological petrology and the archaeometry of lithic materials. Archaeometry 20(2):194-215

Sheppard PJ, Irwin GJ, Lin SC, McCaff rey CP (2011) Characterization of New Zealand obsidian using PXRF. JArchaeol Sci 38:45-56

Sieveking GDG, Craddock PT, Hughes MJ, Bush P, Ferguson J (1970) Characterization of prehistoric flint mine products. Nature 228(5268):251-254

Sieveking GDG, Bush P, Fergusson J, Craddock PT, Hughes MJ, Cowell MR (1972) Prehistoric flint mines and their identifications as sources of raw material. Archaeometry 14(2):151-176

Sieveking GDG, Hart MB (eds) (1986) The scientific study of flint and chert. Proceedings of the Fourth International Flint Symposium Held at Brighton Polytechnic 10-15 April 1983. Cambridge University Press, Cambridge

Tarantini M (2005) Archeologia mineraria della selce nel Gargano. Nuove ricerche. In: Gravina A (ed) Atti $25^{\circ}$ Convegno Nazionale sulla Preistoria, Protostoria, Storia della Daunia (S. Severo 2004), pp $43-55$

Tarantini M, Eramo G, Monno A, Muntoni IM (2016) Gargano Promontory chert: mining practices and archaeometric characterisation. Séances de la Société préhistorique française 5:249-267

Tarantini M, Galiberti A (2011) Le miniere di selce del Gargano VI-III millennio a.C. Alle origini della storia mineraria europea. All'Insegna del Giglio, Firenze

Tarantini M, Galiberti A, Mazzarocchi F (2011) Prehistoric flint mines of the Gargano: an overview. In: Capote $\mathrm{M}$ et al (eds) Flint mining and quarrying techniques in pre- and protohistoric times. Archaeopress, Oxford, pp 253-263

Tucker ME (2001) Sedimentary petrology. In: An introduction to the sedimentary rocks. Blackwell Scientific Publications, Oxford Boston

Turq A (2005) Réflexions méthodologiques sur les études de matières premières lithiques. 1 - Des lithothèques au matériel archéologique. PALEO Revue d'archéologie préhistorique 111-132

Tykot RH (2004) Scientific methods and applications to archaeological provenance studies. In: Martini M, Milazzo M, Piacentini M (eds) Proceedings of the International School of Physics "Enrico Fermi", Course CLIV. IOS Press, Amsterdam, pp 407-432

Valensi L (1960) De l'origine des silex protomagdaléniens de l'abri Pataud, Les Eyzies. Bulletin de la Société Préhistorique Française 52(9-10):584-597

Volterra E, Palmieri AM, D'Ottavio F (2002) Provenance studies of prehistoric chert from the Gargano mines (Puglia, Italy): sources characterisation. In: Jerem E, Biró KT (eds) Archaeometry 98. Proceedings of the 31 st Symposium (Budapest 1998). BAR International Series 1043, vol. II. Archaeopress, Oxford, pp 829832

Wickham H (2016) ggplot2: elegant graphics for data analysis. SpringerVerlag, New York

Zappaterra E (1994) Source-rock distribution model of the periadriatic region. AAPG Bull 78(3):333-354

Publisher's note Springer Nature remains neutral with regard to jurisdictional claims in published maps and institutional affiliations. 